\title{
HEAT FLUX ESTIMATION \\ FOR MATERIALS WITH PYROLYSIS AND ABLATION
}

\author{
S. Alestra, Simulation Information Technology and Systems Engineering, EADS IW, \\ 18 Rue Marius Terce, Campus Engineering, 31000 Toulouse, France \\ J. Collinet, Re-entry Systems \& Technologies, EADS Astrium, \\ 66, route de Verneuil, BP2, 78133 Les Mureaux Cedex, France \\ F. Dubois, Department of Mathematics, Conservatoire National des Arts et Métiers, \\ 292, rue Saint Martin, F-75141 Paris Cedex 03, France
}

stephane.alestra@eads.net; jean.collinet@astrium.eads.net; duboisf@cnam.fr

\author{
44ème Colloque AAAF d'Aérodynamique Appliquée \\ Nantes, 23-24-25 mars 2009 \\ Maîtrise des incertitudes en aérodynamique expérimentale et numérique
}

15/12/2009, to be published in Int. J. Engineering Systems Modelling and Simulation, 2010

\begin{abstract}
In the development of future atmospheric reentry vehicles, the heat shield (and its sizing) is one of the major challenges of the design. It is therefore important to know precisely aerothermal heat fluxes encountered during the reentry, and the associated uncertainties. Identification of these stresses is possible only from indirect measurements, usually based on thermocouples located directly in the thermal protection. In this context, Astrium has developed since many years a one-dimensional tool to evaluate the heat loads on pyrolysable and ablative materials. An inverse problem is formulated to restore the heat flux encountered in re-entry problems, from temperature measurements made inside the material. We minimize the difference calculation / measurement with optimal control techniques (definition of a Lagrangian with adjoint and gradient techniques, with a quasi-Newton algorithm). On-ground and in-flight tests applications are presented, and first encouraging results using the automatic differentiation tool TAPENADE, developed at INRIA.
\end{abstract}

Keywords: inverse problem, ablation, pyrolysis, thermal protection, reentry, optimal control, adjoint, gradient, optimization, automatic differentiation.

\section{Biographical Notes}

Stéphane ALESTRA received the Ph. D. degree of Applied Mathematics (University of Paris) in 1997. He is an engineer at EADS IW since 1998 in the applied mathematics group. He is specialized in numerical modelling and development of scientific algorithms. His fields of expertise are propagation of waves, Finite Difference in Time Domain method, Inverse method for optimal control, Optimisation, and modelling of multi-physics problems (acoustics, thermics, mechanics, electromagnetism, trajectory). He is actually in charge of the inverse techniques and optimisation activity at EADS IW Toulouse.

Jean COLLINET joined EADS Astrium in 2000 as R\&D engineer to work on CFD tools used for steady and unsteady phases related to launchers' development. Specialist in fluid mechanics, he investigated turbulence modeling, gases thermochemistry or numerical techniques in CFD and meshing. He was involved in programs such as Ariane5, M51, ARD Postflight. In 2005, he joined the re-entry aerothermodynamics team and experienced modeling of thermal protection systems, aerodynamics of re-entry vehicles and on-ground and in-flight tests. In 2009, he decided to join propulsion systems department, to design new spacecrafts and improve numerical simulations of rocket motors.

François DUBOIS was born in Tours (France) in 1958. He was student of the Ecole Normale Supérieure (Paris) and obtained a PhD in Applied Mathematics at the University of Paris 6 in 1988. He spent six years for the development of computational fluid dynamics in aerospace industry at «Aerospatiale Les Mureaux ». Since 1994, he is «Professeur des Universités » in Mathematics at the Conservatoire National des Arts et Métiers (Paris) and scientific advisor for Astrium Space Transportation and EADS France Research Center. He is member of Société Mathématique de France and Association Française de Science des Systèmes. 


\section{Nomenclature}

A $\quad=$ Frequency factor in pyrolysis $\left(\mathrm{s}^{-1}\right)$

$\mathrm{B}=$ Activation temperature in pyrolysis $(\mathrm{K})$

$C_{p} \quad=$ Heat capacity $(\mathrm{J} / \mathrm{kg} / \mathrm{K})$

$\mathrm{d} / \mathrm{dinf}=$ Density ratio (local over upstream)

$d f \quad=$ First derivative of $f$ function

$d^{2} f=$ Second derivative of $f$ function

$d_{r} \quad=$ Descent direction in optimization iteration $\mathrm{r}$

$\mathrm{e}=$ Thickness of the one-dimensional slab $(\mathrm{m})$

$\mathrm{F}=$ Operator of direct evolution problem

$F_{v} \quad=$ Pyrolysis gas formation heat $(\mathrm{J} / \mathrm{kg})$

$\mathrm{f}=$ Discrete operator of evolution problem

$H_{c} \quad=$ Pyrolysis gas combustion heat $(\mathrm{J} / \mathrm{kg})$

$H_{r} \quad=$ Hessian approximation at optimization $r$ iteration

$H_{v} \quad=$ Ablation heat $(\mathrm{J} / \mathrm{kg})$

$h_{g} \quad=$ Pyrolysis gas enthalpy $(\mathrm{J} / \mathrm{kg})$

$h_{r} \quad=$ Athermanous enthalpy $(\mathrm{J} / \mathrm{kg})$

$h_{w} \quad=$ Surface enthalpy $(\mathrm{J} / \mathrm{kg})$

Inst $t^{n+1}=$ Solver Program instruction at time $(\mathrm{n}+1)$

$J(p) \quad=$ Cost function

$\mathrm{Kn} \quad=$ Knudsen number

$\mathrm{Kp} \quad=$ Pressure coefficient $: \mathrm{Kp}=\left(\mathrm{P}-\mathrm{P}_{\text {upstream }}\right) / \mathrm{P}_{\mathrm{dyn}}$

$L=$ Lagrangian multiplyer

$\mathrm{n}=$ Time iteration

$\dot{m}_{c} \quad=$ Ablation mass flow rate $\left(\mathrm{kg} / \mathrm{m}^{2} / \mathrm{s}\right)$

$\dot{m}_{g} \quad=$ Pyrolysis gas mass flow rate $\left(\mathrm{kg} / \mathrm{m}^{2} / \mathrm{s}\right)$

$\mathrm{K}=$ Number of $1 \mathrm{D}$ Grid points

$\mathrm{k}=$ Space index

$\mathrm{N}=$ Number of time iterations

$N_{o p} \quad=$ Number of optimizer iterations

$\mathrm{n} \quad=$ Time index

$P A \quad=$ Mechanical erosion coefficient $\left(\mathrm{m}^{2} . \mathrm{s} / \mathrm{kg}\right)$

$P B=$ Normal constraint coefficient

$\mathrm{p} \quad=$ Parameter

$p^{n} \quad=$ Parameter value at time $\mathrm{n}$

$p_{\text {opt }} \quad=$ Optimal parameter

$\mathrm{Q}=$ Heat flux $\left(\mathrm{W} / \mathrm{m}^{2}\right)$

$q_{r} \quad=$ Parameter at optimizer iteration $\mathrm{r}$

$\mathrm{r}=$ Optimizer iteration indice

$\mathrm{ReL} \quad=$ Reynolds number

$\mathrm{St} \quad=$ Stanton (adimentional heat flux)

$\mathrm{s} \quad=$ Ablation variable $(\mathrm{m} / \mathrm{s})$

$s^{n} \quad=$ Ablation variable computed at time $\mathrm{n}(\mathrm{m} / \mathrm{s})$ $\dot{s}_{\text {meca }}=$ Mechanical Recession rate $(\mathrm{m} / \mathrm{s})$

$\dot{s}_{\text {chem }}=$ Chemical Recession rate $(\mathrm{m} / \mathrm{s})$

$\dot{s}_{h y} \quad=$ Hydroerosion Recession rate $(\mathrm{m} / \mathrm{s})$

$\mathrm{T}=$ Temperature $(\mathrm{K})$

$T_{o p t} \quad=$ Optimal Temperature (at optimal $\left.\mathrm{p}\right)(\mathrm{K})$

$T_{0} \quad=$ Reference initial temperature $(\mathrm{K})$

$T_{k}^{n} \quad=$ Temperature computed at time $\mathrm{n}$, point $\mathrm{m}(\mathrm{K})$

$T_{E} \quad=$ Mechanical erosion fictitious temperature (K)

$T_{r} \quad=$ Equivalent temperature $(\mathrm{K})$

$T_{w} \quad=\quad$ Surface temperature $(\mathrm{K})$

$\mathrm{t}=$ Time $(\mathrm{s})$

$t_{f} \quad=$ Final time $(\mathrm{s})$

$\mathrm{W}=$ Continuous Direct state variable: temperature \& ablation

$\mathrm{w}=$ Discrete Direct state variable: temperature \& ablation

$w^{n} \quad=$ Direct state variable at time iteration $\mathrm{n}$

$x=$ Sensor position $(\mathrm{m})$

$x_{0} \quad=$ Sensor position $(\mathrm{m})$

$\mathrm{z} \quad=$ Hessian intermediate function

$\alpha_{0}$

$\beta_{n} \quad=$ Gear coefficient at time iteration $\mathrm{n}$

$\gamma \quad=$ heat capacity ratio

$\Delta t \quad=$ Time step (s)

$\varepsilon \quad=$ Total Emissivity

$\eta_{1} \quad=$ Pyrolysis gas blocking factor

$\eta_{2}=$ Ablation gas blocking factor

$\theta=$ Measured temperature $(\mathrm{K})$

$\theta_{m}^{n} \quad=$ Measured temperature at time $\mathrm{n}$, point $\mathrm{m}(\mathrm{K})$

$\lambda=$ Thermal conductivity $(\mathrm{W} / \mathrm{m} / \mathrm{K})$

$\mu \quad=$ Descent coefficient for optimizer

$\mu_{r} \quad=$ Descent coefficient at optimizer iteration $\mathrm{r}$

$\xi \quad=$ Reduced scaled abscissa

$\rho=$ Specific Mass $\left(\mathrm{kg} / \mathrm{m}^{3}\right)$

$\rho_{c} \quad=$ Charred material densities $\left(\mathrm{kg} / \mathrm{m}^{3}\right)$

$\rho_{v} \quad=$ Virgin material densities $\left(\mathrm{kg} / \mathrm{m}^{3}\right)$

$\sigma \quad=$ Stefan-Boltzmann constant $\left(\mathrm{W} / \mathrm{m}^{2} / \mathrm{K}^{4}\right)$

$\tau \quad=$ Mechanical erosion fictitious constraint $\left(\mathrm{N} / \mathrm{m}^{2}\right)$

$\varphi=$ Discrete Adjoint state variable: temperature $\&$ ablation

$\varphi^{n+1 / 2}=$ Adjoint state variableat time $\mathrm{n}+1 / 2$ 


\section{Introduction}

In the field of atmospheric re-entry, the missions can be quite different depending on the goal: reentry from a low orbit, with or without crews, exploration of other planets, with or without return of samples or military exercises. The range of speeds encountered is very large and may in some cases exceed $10 \mathrm{~km} . \mathrm{s}^{-1}$.

A key to the success of such applications lies in the conception and design of thermal protection systems of the spacecraft used. The levels of very high aerothermal fluxes encountered have a direct impact on the mass balance, this mass being in general a parameter to optimize in space applications.

Thus, knowledge, identification, prediction of heat flux is of considerable industrial interest, combined with significant challenges in terms of feasibility of certain projects, very constrained by mass and equipement issues.

Unfortunately, these fluxes are difficult to measure in flight and their identification is usually possible only by indirect methods, based on measurements of temperature in heat shields. This problem can be illustrated among others by the test flight of the $\mathrm{ARD}^{1-3}$ (Atmospheric Reentry Demonstrator).

One of the difficulties inherent in the reentry flight test is to distinguish between uncertainty related to the estimation of aerothermal fluxes and uncertainties related to the behavior of the materials used (eg through control of thermal properties of the latter). In this study, the degradable material (ablation and pyrolysis phenomena of thermal protection) is supposed to be well known, and the analysis is limited to the identification of aerothermal fluxes and uncertainties.

Many works also deal with ablative and pyrolysable heat shields behaviour problems ${ }^{4-6}$. Validations on ground with "high enthalpies tests" such as plasma torch ${ }^{7}$ help to better understand these problems and to carry out the design of various reentry vehicles ${ }^{8-12}$.

First, the various sources of uncertainty associated with the evaluation of flux will be illustrated. Many authors have addressed the issue of restitution of conductive flux by inverse method, from measurements of température ${ }^{13-17}$. Recently, three-dimensional methods on non-degradable materials have been successfully developed $^{18}$.

Secondly, an inverse method is described for the estimation of evolutionary time heat fluxes on an ablative and pyrolysable material submitted to significant stress and equipped with temperature measurements (thermocouples) located inside the material.

This inverse method leads to usual algorithms of optimization. Thus, a formulation issued from optimal control techniques (involving a Lagrangian, an adjoint formulation, and a calculation of gradients) is proposed ${ }^{19}$. These techniques have been used in the past, to formulate and solve several inverse problems with industrial applications of EADS (European Aeronautics Defense and Space Company) ${ }^{20-21}$.

The numerical tool "Monopyro" developed by EADS Astrium Les Mureaux ${ }^{22-23}$ allows to adress, in direct or reverse mode, the one dimensional heat process in thermal protection. This tool takes into account many physical phenomena associated with high fluxes, such as ablation (treatment of a mobile interface), pyrolysis (complex chemistry inside material).

The validation of the tool described below will be presented through numerical tests performed in configurations from simple (pseudo measurements on an inert material) to the most sensitive (pseudo noise measurements on pyrolysis and ablative material), before considering cases that are more operational, such as heat flux restitution during the ARD flight or numerically more severe tests of materials in a plasma torch.

During the work presented below, the use of a tool for automatic differentiation, to generate automatically the reversed code, has also been successfully tested on a simple case first.

This application allows considering in the future a more systematic use of automatic differentiation tools, which may also provide valuable assistance in the calculation of uncertainties.

\section{Flux Uncertainties}

Aerothermal heat fluxes are one of the key parameters for the sizing of thermal protections used in the field of atmospheric re-entry. It is thus crucial for the industry to control the uncertainties associated with the evaluation of these sollicitations

The complex physical phenomena involved in the descent phase in the atmosphere and their effects on thermal and mechanical stresses must be considered at best. Thus, the heat flux encountered are very sensitive to the following phenomena: rarefaction effects at high altitude, so-called "real gas" effects linked to the gas chemistry of the air at high temperature (creation of an air plasma around the vehicle), changes of aerodynamic shape related to the removal of insulation, blowing effects of the degassing heat shields (pyrolysis), surface roughness also related to the degradation of materials.

The factors of uncertainty usually associated with these flows' knowledge corresponds to the specific flows encountered during these missions (reactive flows, rarefied or not, hypersonic regime, laminar-turbulent transition), associated with specific flow interactions with the thermal protection (ablation, roughness, blowing protections). Margins policy associated with these uncertainties can then ensure a good behavior of these vehicles.

The first specificity of the reentry is the variability of flow regime encountered along a reentry trajectory. The Knudsen number $(\mathrm{Kn})$, which is the ratio of the mean free path over the characteristic dimension of the spacecraft 
can be introduced to determine this flow regime. At very high altitude, the mean free path of atoms and molecules is big compared with the length of the vehicle, the flow is said to be in free molecular regime $(\mathrm{Kn}>>1)$.

At low altitude, the mean free path becomes negligible compared with the characteristic dimensions of the vehicle and we have the continuous regime $(\mathrm{Kn} r<1$, the flow can then be described by the Navier-Stokes equations). Between these two extreme regimes, the mean free path and size of the vehicle are in a moderate ratio, and the flow is said rarefied (typically it is necessary to solve the Boltzman equation to describe the flow).

If it can be showed in some wind tunnel, the rarefaction effect remains difficult to quantify because the wind tunnel test must have accurate measurements to obtain very accurate results. It is also possible to capture these effects by DSMC (Direct Simulation Monte Carlo) simulations. An example of simulations on the reentry vehicle IXV (Intermediate eXperimental Vehicle) is proposed in Figures 1,2 and 3, which correspond to altitudes of 117,100 and $94 \mathrm{~km}$.

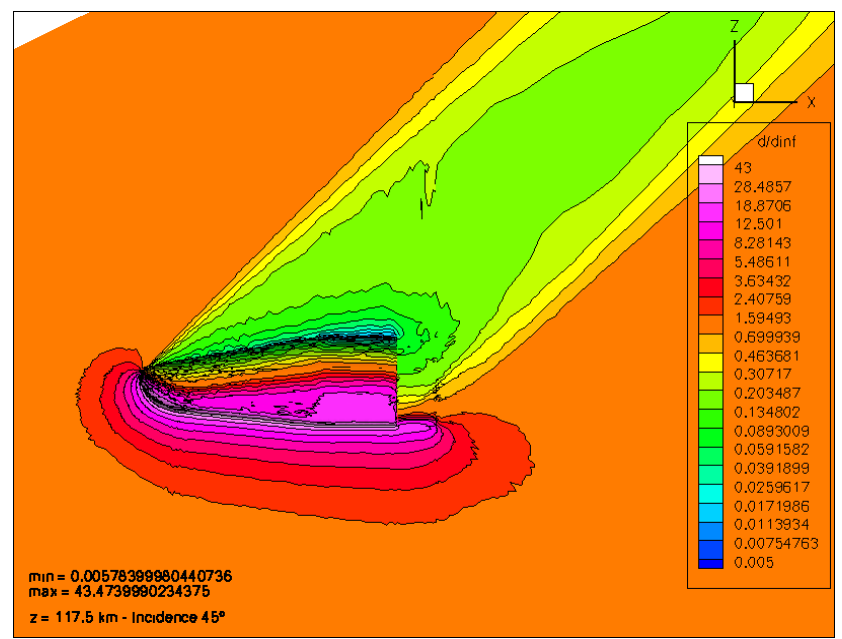

Figure 1: IXV - Density ratio in rarefied regime $\mathrm{Z}=117 \mathrm{~km}$, angle of attack $=45^{\circ}$

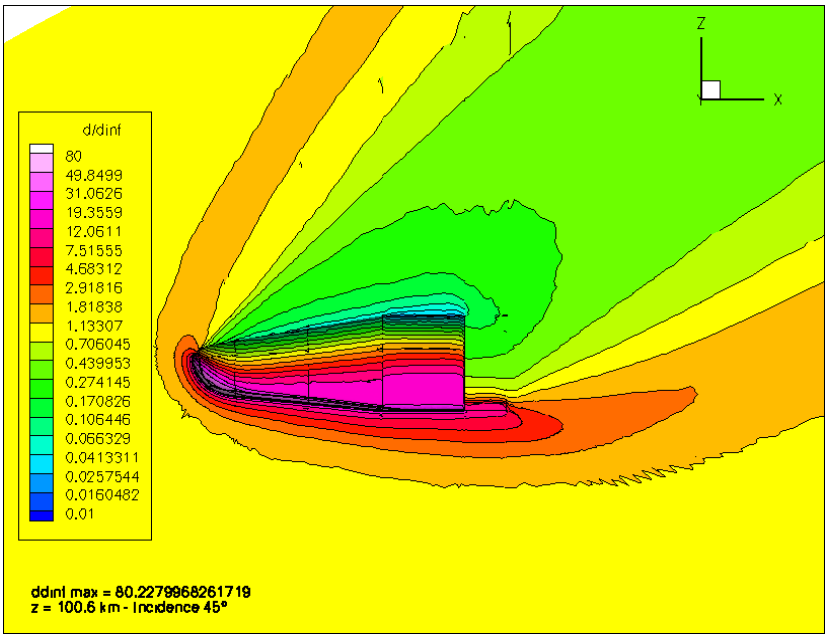

Figure 2: IXV - Density ratio in rarefied regime $\mathrm{Z}=100 \mathrm{~km}$, angle of attack $=45^{\circ}$

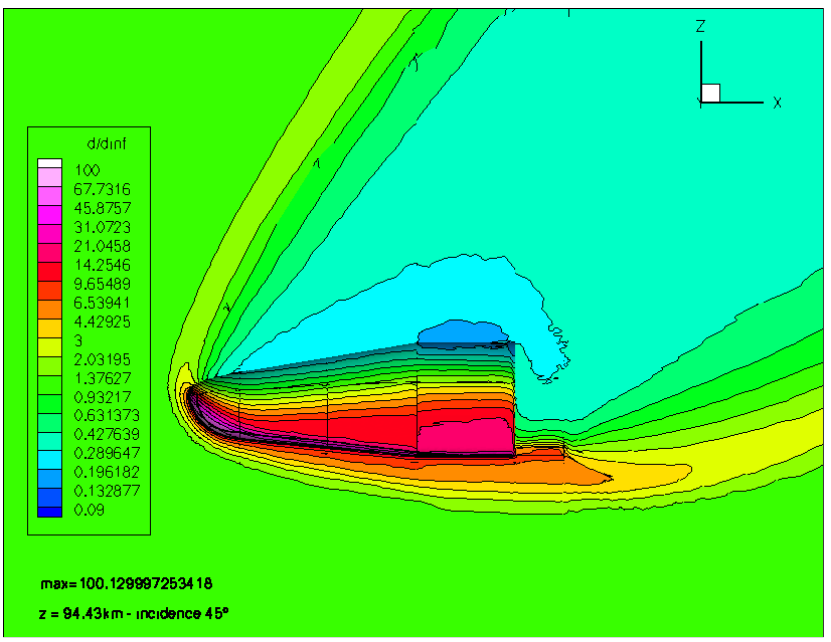

Figure 3: IXV - Density ratio in rarefied regime $\mathrm{Z}=94 \mathrm{~km}$, angle of attack $=45^{\circ}$

In continuous regime, it is then necessary to distinguish the laminar regime and the turbulent regime, for which fluxes become stronger. The uncertainties on the fluxes for hypersonic flows encountered in the process of re-entry are often linked to the chemical phenomena involved around the vehicle. The speed of the vehicle is high enough to produce a detached shock wave upstream. The air is then subject to such a temperature that it can be ionized (or simply dissociated into single atoms of oxygen and nitrogen).

Fluxes on the vehicle depend on the chemical kinetics, with very different effects depending on the size of the vehicle (chemistry close to equilibrium or frozen depending on the case). Uncertainties in terms of calculation can then be quantified through studies of the sensitivity of influent parameters, the hypersonic wind 
tunnel tests also participating to the reduction of uncertainty as much as possible. Such an approach has been implemented in the program IXV ${ }^{24}$. Figure 4 shows, for instance, the influence on the flux of the chemical kinetic model used to model the air, the main differences being observed around the nose and flaps of the IXV.

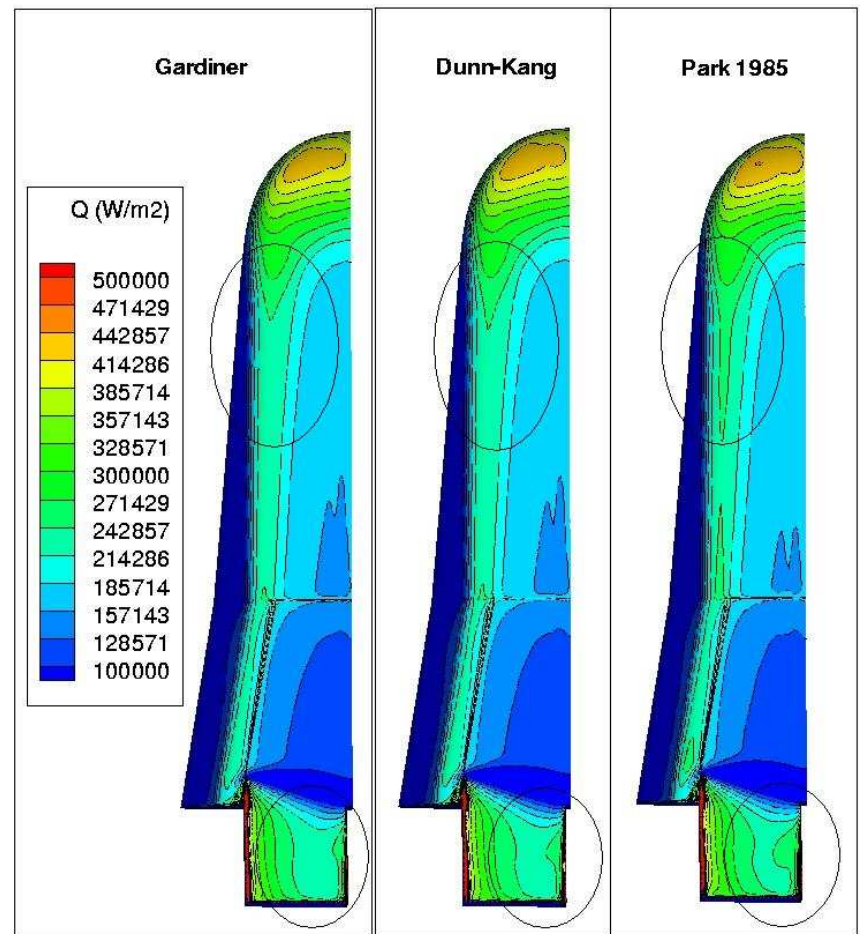

Figure 4: IXV - Distributions flow at Mach 25 for different models of chemical kinetics

Similarly, Figure 5 shows the influence on the heat flux, at Mach 25, of the model used to simulate the transport properties of air (viscosity and conductivity).

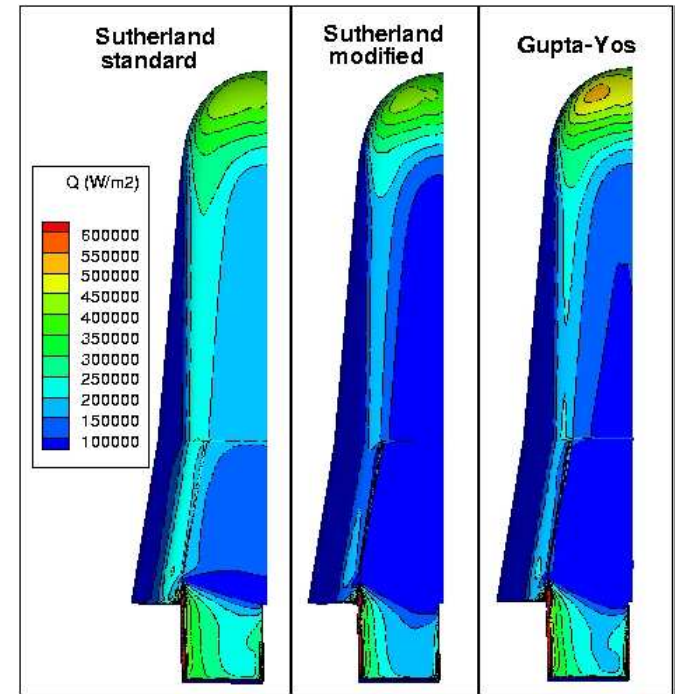

Figure 5: IXV - Distributions of flux at Mach 25 for different transport properties behaviour
The reactivity of the wall, mainly due to the nature of the latter, is also an important source of uncertainty in the flux, as shown in Figure 6, which compares the case of a fully catalytic wall (total recombination of oxygen atoms and of nitrogen) to the case of non-catalytic wall (zero species gradient on the wall)

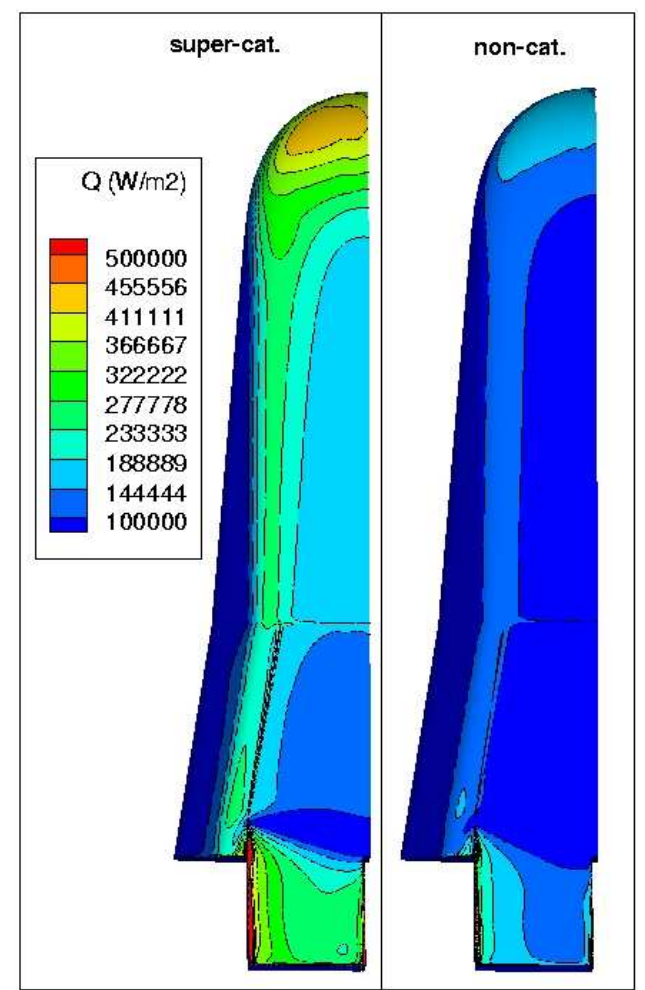

Figure 6: IXV - Distributions of flux at Mach 25 for different wall reactivities

Nevertheless, the air can also be regarded as a perfect gas when Mach numbers are smaller, the difference with more complex models can then be quantified, as shown in Figure 7 which shows a Mach 17.7 evaluation in the case of perfect gas, compared to the case of equilibrium chemistry model. The difference is significant mainly on the flaps partly because the flow is (for the values of the flap deflection considered) at the limit of the laminarturbulent transition in this area, so the models are all the more sensitive and difficult to calibrate. 


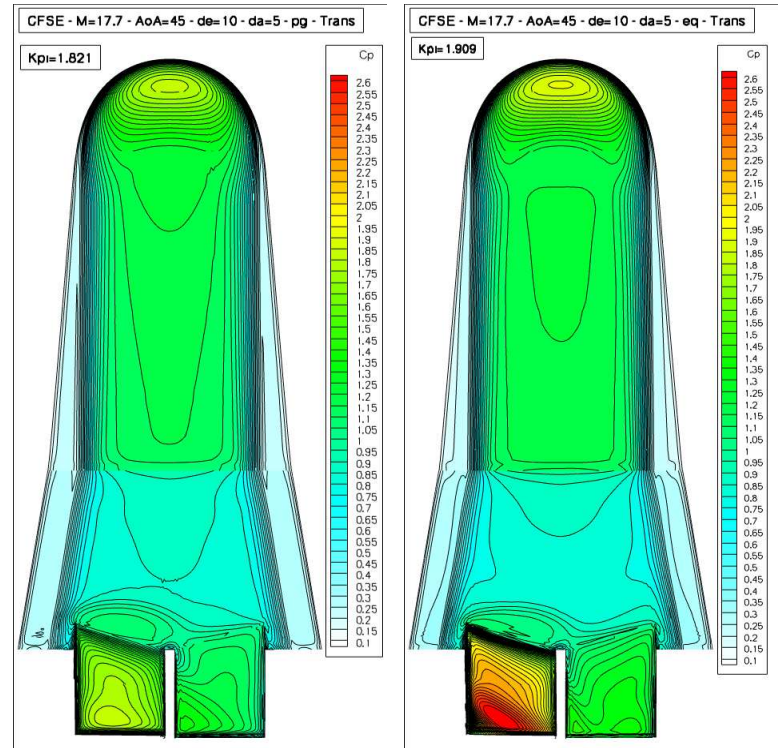

Figure 7: IXV - Kp Distributions Mach 17.7

perfect gas $(\gamma=1.4$, left) and equilibrium (right)

Finally, Figure 8 illustrates the IXV studies by means of wind tunnel tests (here $\mathrm{H} 2 \mathrm{~K}$ ) or by simulation (NavierStokes) used to characterize the laminar-turbulent transition and the values of turbulent fluxes.

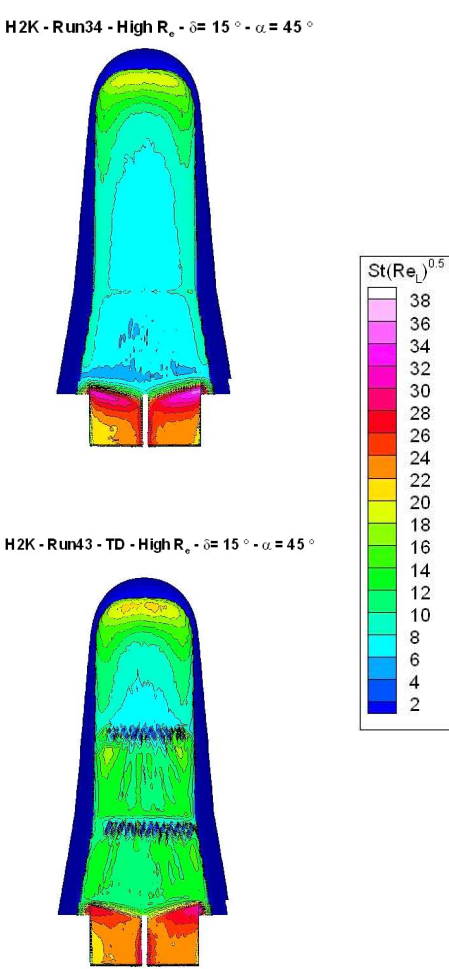

H2K NS - High $R_{e}-\delta=15^{\circ}-\alpha=45^{\circ}$ - Transitiona

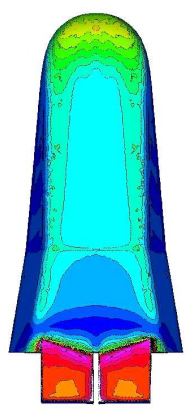

H2K NS - High $R_{\mathrm{e}}-\hat{\delta}=15^{\circ}-\alpha=45^{\circ}$ - Turbulent

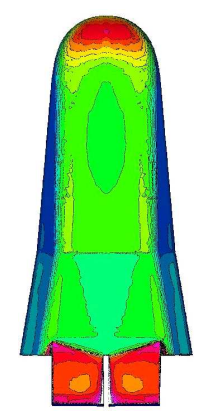

Figure 8: IXV - Distributions of adimensional flux in transition regime (top) and turbulent (lower)

obtained by tests (left) and simulated (right)

The uncertainties in turbulent regime are still fairly high on the fluxes, particularly in terms of simulations, which provide very different flux results depending on the areas of the flow considered and according to the turbulence models used. Turbulence contributes much to the uncertainties associated with aerothermal fluxes and remains a critical parameter in terms of sizing and success of reentry missions.

Let us mention also that for some fast reentry to Earth or some reentries in exotic atmospheres, the radiation of the plasma around the body must be taken into account in assessing the flux on the thermal protection (the reentry, not shown here, of the probe Huygens in the atmosphere of Titan is a good example).

Other uncertainties factors come from interactions with the thermal protection material. To masterize the reentry of vehicles, it is necessary to quantify the effects related to the removal of the thermal protection (change of external shape, degassing), its pyrolysis (effects of flow blocking) or the roughness which develop at the surface of the material when ablating.

To evaluate these contributions in the assessment of uncertainties, to establish at the end, an objective of margins for spacecrafts, Astrium has conducted a number of experimental and simulations campaigns. The effects of change in form can be better apprehended. Figures 9 and 10 offer an example of Navier-Stokes simulations made on a configuration of test material in a plasma torch. A test cylinder, specimen of thermal protection material is put in the jet of the plasma torch under conditions representative of flight stresses.

Following the upstream pressure in the torch (high point or low point), the flow on the specimen to be tested is significantly changed. The same structures can be both observed experimentally (fast camera at top) and numerically simulated (iso-Mach contours at the bottom). These simulations, coupled with a thermal ablative material, allow a better prediction of the effects of changing shapes on ground and in flight.

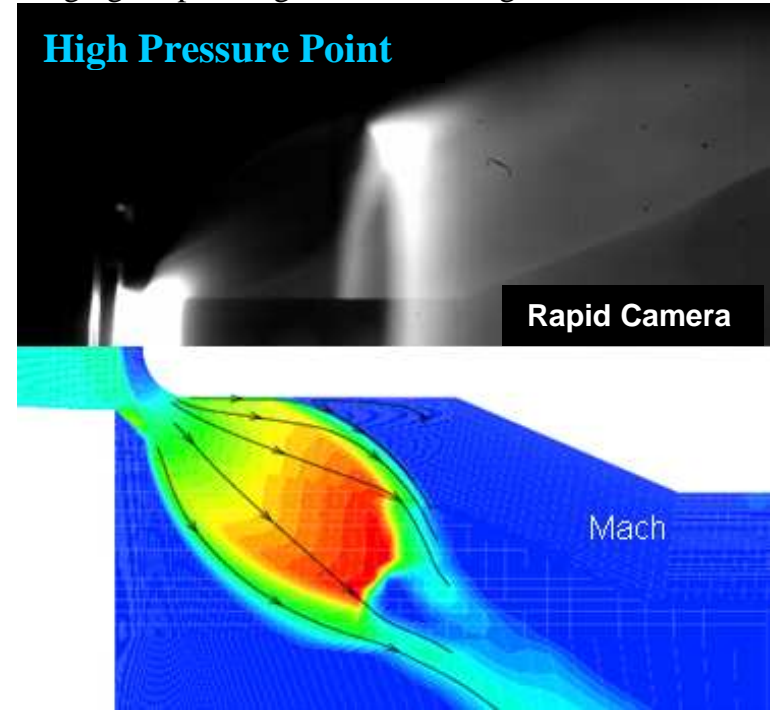

Figure 9: Test of materials with plasma jet high pressure upstream 


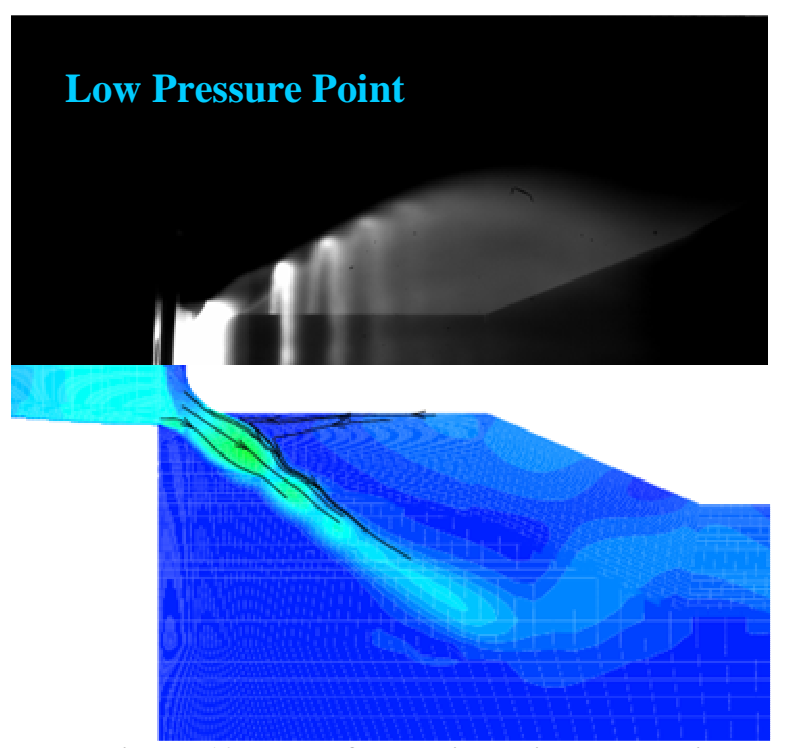

Figure 10: Test of materials with plasma jet low pressure upstream

Wind tunnel tests at ONERA (R2CH Meudon, cf. Figure 11) were used to estimate, also on the flux, the separated effects of roughness on the surface of thermal protection, and of an emission of wall gases.

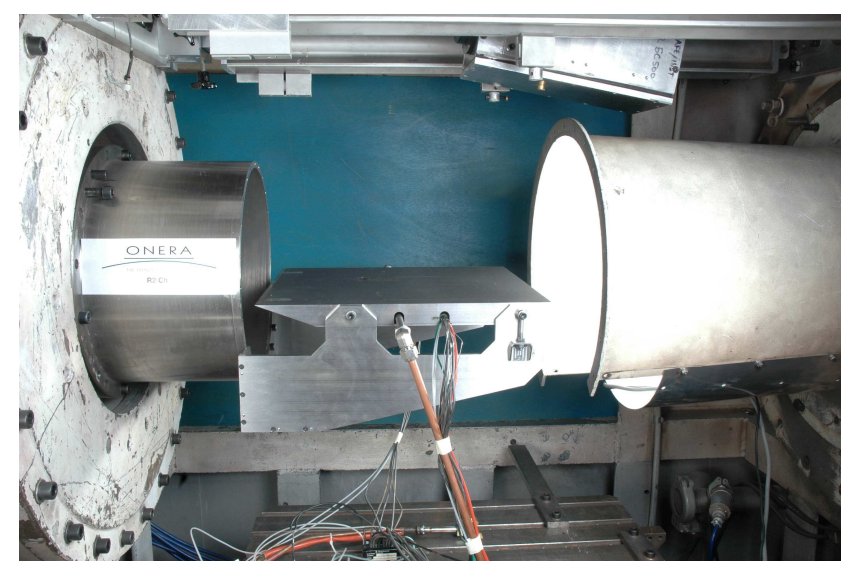

Figure 11: R2CH Test - Mounting

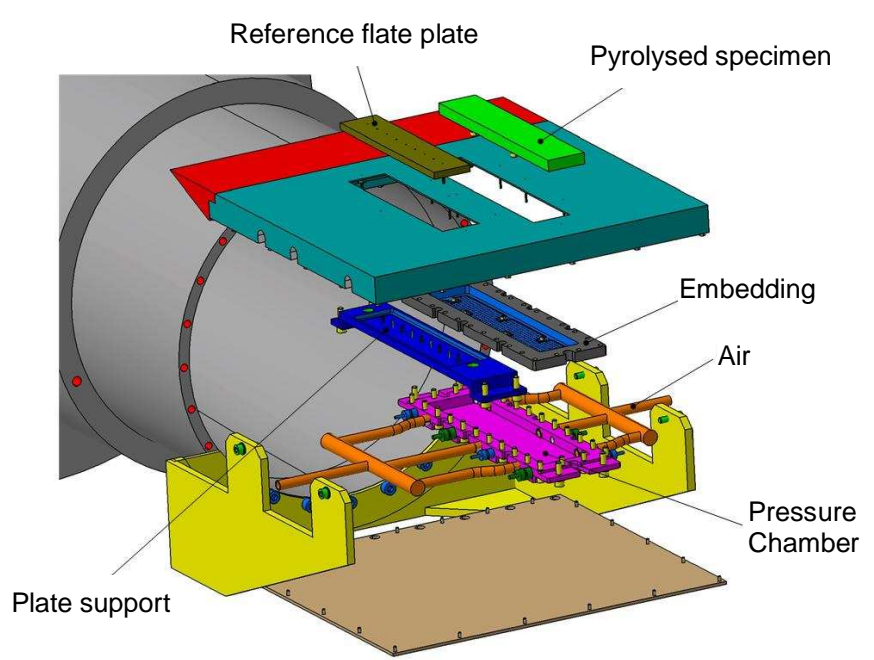

Figure 12: R2CH Test - Schematic of mounting

The test mounting (see Figures 11 and 12) allows to compare the fluxes on a reference flat plate and on a specimen of the tested material previously pyrolysed (and rough) in conditions representative of flight. Figure 13 shows a visualization of the flow (Mach 5), which shows the effect of roughness, an infrared camera also providing a flux along the thermal protection sample and on the reference plate (see Figure 14). The flux profile along the pyrolysed specimen is very well correlated with the surface roughness profile.

In Figure 14, the comparison with the reference plate enlights an effect of wall temperature (the thermal protection being much more isolating than the witness plate) modifying the boundary layer.

Part of the difference probably also reflects the uncertainties on the thermal properties of the pyrolysable material (impacting on the experimental heat flux restitution from the infrared measurements).

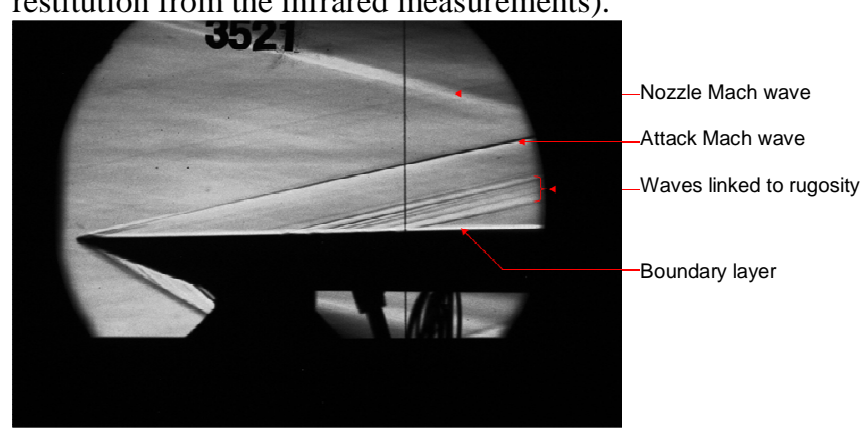

Figure 13: Strioscopic view - Mach 5 


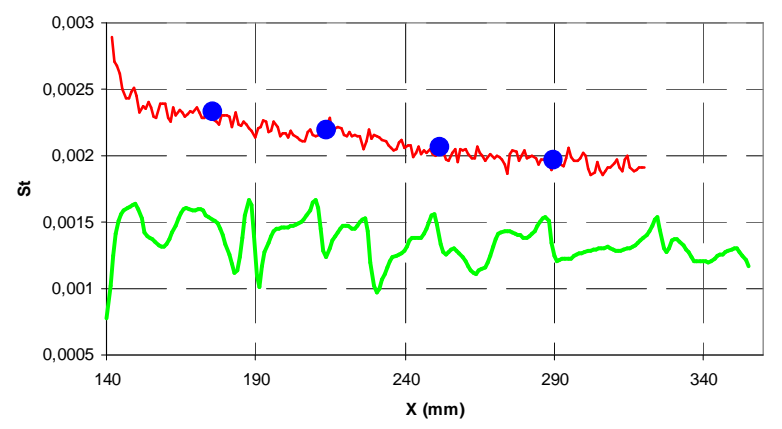

Figure 14: Comparison of adimensional IR flux smooth $($ red) $/$ rough $($ green) $($ blue $=$ thermocouples)

A pressurized injection chamber (see Figure 15) also allows injecting air through a porous ceramic specimen or directly through the thermal protection material.

Then the test can give, for different air mass flows injected, the blocking effect on the flux. Figure 16 provides a comparison of the heat fluxes obtained for two injection ratio and without injection. The expected blocking effect is observed.

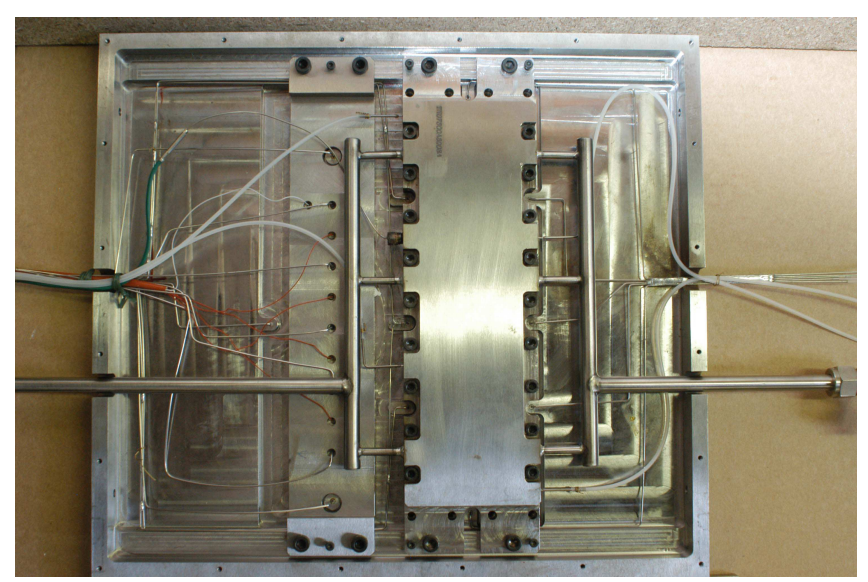

Figure 15: Injection system in WTT model

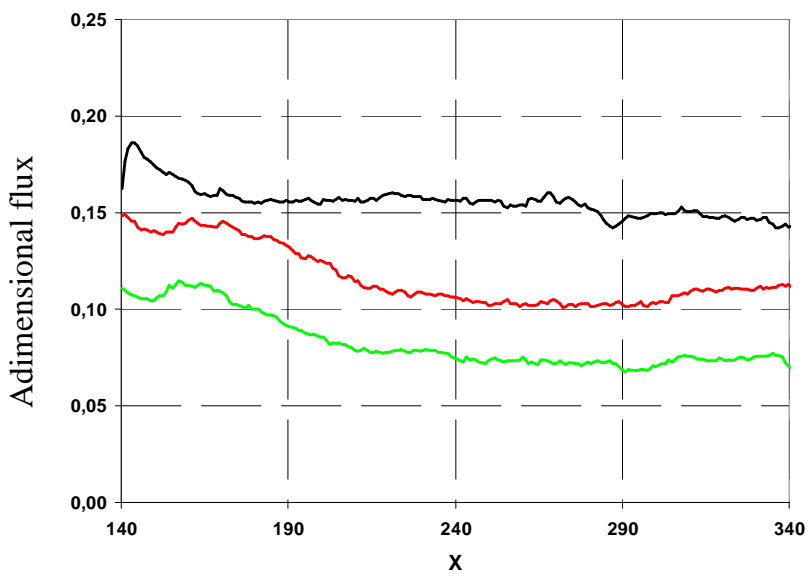

Figure 16: Comparison of adimensional fluxes (black = no blowing, red $=$ low blowing, green $=$ high $)$
These different effects on the flux being mainly quantified on the ground, the difficulty is to transpose them in flight. The validation of the models selected is difficult because it is necessary to distinguish all the effects, which are sometimes conflicting.

It is in this context that Astrium has developed the method described below, which allows to restitute during the flight, from temperature measurements (thermocouples located in the thermal protection), the "efficient" flux as seen by the material (by assuming its thermal and ablative properties well known). One aspect of the exploitation of measurements consists in, starting from this knowledge of efficient flux seen by the material, to separating, quantifying and explaining the different effects seen in flight.

\section{Direct problem}

\section{Continuous equations}

A transient one-dimensional thermal problem with one moving boundary (ablative surface) has been developed and used at EADS Astrium ${ }^{25,26}$ to model complex chemical processes of simultaneous heating, pyrolysis, ablation and thermal degradation behaviour of ablative materials. We briefly present the direct model used.

Internal energy balance (for pyrolysable ablative material) :

The internal energy balance is a transient thermal conduction equation with additional pyrolysis terms

$\rho C_{p} \frac{\partial T}{\partial t}=\frac{\partial}{\partial x}\left(\lambda \frac{\partial T}{\partial x}\right)+\left[F_{V}+h_{g}-\int_{T 0}^{T} A_{1} d T\right] \frac{\partial \rho}{\partial t}+\frac{\partial\left(\dot{m}_{g} h_{g}\right)}{\partial x}(1)$ with $\mathrm{x}$ the abscissa, $\mathrm{t}$ the time, $T(\mathrm{x}, \mathrm{t})$ the temperature, $\rho(\mathrm{x}, \mathrm{t})$ the specific mass, $C_{p}$ the heat capacity, $\lambda$ the thermal conductivity, $\dot{m}_{g}$ the pyrolysis gas mass flow rate, $h_{g}$ the pyrolysis gas enthalpy, $A_{1}$ a constant, $F_{v}$ the pyrolysis gas formation heat. The rate of storage of sensible energy is balanced by the net rate of thermal conductive heat flux, the pyrolysis energy-consumption rate and the net rate of energy convected by pyrolysis gas.

Pyrolysis with internal decomposition modelled via a first-order rate process based on the Arrhenius equation

The evolution of specific mass is given by (2):

$$
\frac{1}{\rho_{v}} \cdot \frac{\partial \rho}{\partial t}=-\left(\frac{\rho-\rho_{c}}{\rho_{v}}\right)^{n p} \cdot A \cdot e^{-\frac{B}{T}}
$$


$\rho_{c}$ and $\rho_{v}$ are the charred and virgin material densities, $A$ the frequency factor in pyrolysis, $B$ the fictitious temperature in pyrolysis, $n p$ the order of the reaction. More complex pyrolysis models can be used, for instance as proposed in literature ${ }^{4}$.

Internal decomposition converts some of the solid into pyrolysis gas. The pyrolysis gas mass flux is related to the decomposition by the simple mass balance:

$$
\frac{\partial \rho}{\partial t}=\frac{\partial \dot{m}_{g}}{\partial x}
$$

The surface recession : we denote by $s$ the abscissa of the moving interface (ablation value), then $\dot{s}$ is the recession rate. This physical process can be splitted in three kinds of ablation:

$$
\dot{S}=\dot{S}_{\text {meca }}+\dot{S}_{c h e m}+\dot{S}_{h y}
$$

The mechanical recession rate is modeled by

$$
\dot{s}_{\text {meca }}=P A\left(\tau+P_{e} P B\right) e^{-\frac{T_{e}}{T_{p}}}
$$

with $T_{E}$ the mechanical erosion fictitious temperature, $\tau$ the mechanical erosion fictitious constraint, $\dot{m}_{c}$ the pyrolysis gas mass flow rate, $P A$ the mechanical erosion coefficient, $P B$ the normal constraint coefficient. The chemical recession rate $\dot{s}_{\text {chim }}=\dot{m}_{c} / \rho$ is most of the time a tabulated value function of $\dot{m}_{g} / \alpha_{0}$, of temperature $\mathrm{T}$ and of pressure $\mathrm{P}$ on the material with $\alpha_{0}(\mathrm{t})$ the convection coefficient, or unblocked convective heat transfer coefficient (unknown for inverse problem), and $\dot{m}_{c}$ the ablation mass flow rate. The hydroerosion recession rate $\dot{s}_{h y}$ variable is also most of the time a tabulated value.

\section{Surface energy balance on the moving boundary:}

The conditions at the hot surface are determined by convective heating and by thermochemical interactions of the surface with the boundary-layer gas. The surface energy balance takes the following form:

$$
\begin{aligned}
& \alpha_{0}\left(h_{r}-h_{w}\right)-\varepsilon \sigma\left(T_{w}^{4}-T_{r}^{4}\right)+\dot{m}_{g}\left[H_{c}-\eta_{1}\left(h_{r}-h_{w}\right)\right] \\
& +\dot{m}_{c}\left[H_{v}-\eta_{2}\left(h_{r}-h_{w}\right)\right]=\lambda \frac{\partial T}{\partial x}
\end{aligned}
$$

with $\eta_{1}$ the pyrolysis gas blocking factor, $H_{c}$ the pyrolysis gas heat combustion, $\dot{m}_{c}$ the ablation mass flow rate, $h_{r}$ the athermanous enthalpy, $h_{w}$ the surface enthalpy, $\eta_{2}$ the ablation gas blocking factor, $H_{v}$ the ablation heat, $\varepsilon$ the total emissivity, $\sigma$ the StefanBoltzmann constant, $T_{w}$ the surface temperature, $T_{r}$ the equivalent temperature. The first term of equation (6) represents the convective heat flux. The second term represents the heat loss by re-radiation of the surface. The third and fourth terms represent the contribution of pyrolysis and ablation gas respectively. The term on the right hand of (6) represents the rate of conduction into the TPS.

We introduce $W=\left(\begin{array}{l}T \\ s\end{array}\right)$ the vector of temperature and ablation, functions of time $t$ and position $x$. Therefore, the direct problem can be represented in condensed vector form by the following system of coupled nonlinear time domain evolution differential equations:

$$
\begin{aligned}
& \frac{d W}{d t}=F(W) \\
& T(x, 0)=T_{0} \quad s(x, 0)=0 \\
& t \in\left[0, t_{f}\right], x \in[s(t), e]
\end{aligned}
$$

where $F(W)$ is a non linear operator and $T_{0}$ the reference initial temperature. The other physical quantities and variables described above are hidden in the formulation of $\mathrm{F}$, and in the linear system coefficients than will result from (7) after spatial and temporal discretization.

\section{Discrete scheme}

Space partial derivatives are computed with a centered finite difference type scheme ${ }^{27}$. The abscissa $x$ belongs to the interval $[s(t), e]$. It is parameterized by a reduced scaled space variable $\xi \in[0,1]$ :

$$
x=(1-\xi) s(t)+\xi e
$$

Then the system (7) is rewritten relatively to the variables $(t, \xi)$. The variable $\xi$ is discretized with the help of $\mathrm{K}$ grid points. This complete set of equations has been solved numerically, for non constant time steps, using a one-dimensional two time steps Gear Scheme, which is second order accurate implicit integration scheme, with the approximation of the time derivative on two contiguous time steps $\Delta t^{n-1 / 2}$ and $\Delta t^{n+1 / 2}$, with the $\beta_{n}$ Gear coefficient ${ }^{28}$ : 


$$
\begin{aligned}
& \left(\frac{d w}{d t}\right)^{n+1} \cong \beta_{n} \frac{w^{n+1}-w^{n}}{\Delta t^{n+1 / 2}}+\left(1-\beta_{n}\right) \frac{w^{n}-w^{n-1}}{\Delta t^{n-1 / 2}} \\
& \beta_{n}=\frac{2 \Delta t^{n+1 / 2}+\Delta t^{n-1 / 2}}{\Delta t^{n+1 / 2}+\Delta t^{n-1 / 2}} \quad 0 \leq n \leq N
\end{aligned}
$$

For simplicity, we explain our method on the implicit Euler scheme with a constant time step $\Delta t$. We define $\mathrm{K}$ the number of one-dimensional grid points, $\mathrm{N}$ the number of time iterations, $\mathrm{k}$ the space index, $\mathrm{n}$ the time index in the numerical scheme, $w=\left(w^{1}, \ldots, w^{N}\right)$ the discrete direct state variables matrix of dimension $(\mathrm{K}+1)^{*} \mathrm{~N}$, with the discrete vector $w^{n}=\left(T_{1}^{n}, T_{2}^{n}, \cdots, T_{K}^{n}, s^{n}\right)$ of dimension $(\mathrm{K}+1), T_{m}^{n}$ the discrete computed temperature at time n, at grid point $\mathrm{m}$, for the $\mathrm{K}$ different points on the grid, $s^{n}$ the discrete computed ablation, at time $\mathrm{n}$. The equation (7) is written at time $(n+1)$ :

$$
\begin{aligned}
& \frac{w^{n+1}-w^{n}}{\Delta t}=f\left(w^{n+1}\right) \\
& w^{0}=0 \quad 0 \leq n \leq N
\end{aligned}
$$

We make a linearization of the equation (10) at time $n$ and after some calculations, we finally obtain a forward time discrete linearized Euler scheme, with initial condition vanishing:

$$
\begin{aligned}
& \frac{w^{n+1}-w^{n}}{\Delta t}=f\left(w^{n}\right)+(d f)\left(w^{n}\right)\left(w^{n+1}-w^{n}\right) \\
& w^{0}=0 \quad 0 \leq n \leq N
\end{aligned}
$$

Note that $f\left(w^{n}\right)$ is a vector $(\mathrm{K}+1)^{*} 1,(d f)\left(w^{n}\right)$ is the linearized square matrix $(\mathrm{K}+1) *(\mathrm{~K}+1)$. To solve the discrete matrix problem, we use an adapted sparse solver $^{29}$. In order to focus on the inverse procedure, we won't develop more in details the expressions of the discrete schemes, as the direct scheme is very complex, due to non linearities (complex chemical physical processes, ablation, pyrolysis), tabulated variables for the physical ablation process, and complex linearizations and discretizations.

\section{Inverse problem}

Inverse problems are concerned with the identification of unknowns and the improvement of the understanding of physical processes quantities which appear in the mathematical formulation of physical problems, by using measurements of the system response.
The inverse problem in this paper is used to estimate time domain surface heat fluxes (convection coefficient), for degradable material (ablation and pyrolysis), on a onedimensional slab of thickness $e$, by using time domain temperature measurements $\theta(t)$ on thermal protection, taken below the boundary surface, at thermocouple position $x_{0}$, during the time interval $0 \leq t \leq t_{f}$, where $t_{f}$ denotes the final time. The inverse problem is reformulated as a minimization problem involving a cost objective functional, through an optimization loop, requiring the computation of derivatives or gradients quantities and adjoint variables (optimal control formulation).

\section{Discrete problem and cost function}

To obtain an accurate numerical approximation of the gradient, the key strategy is to compute the exact gradient of the discretized problem, instead of applying a discretization scheme to the above systems of PDE- $\mathrm{s}^{30}$.

Therefore the best way is to proceed to the derivation of the direct schemes. Let us consider that the time domain content of the unknown heat flux convection coefficient is represented by a vector $p=\left(p^{1}, \ldots, p^{N}\right)$ which is sampled over time, where the subscripts refer to the sampled time. $\mathrm{N}$ is the number of unknowns and time iterations. These sampled values will be the control parameter variables for the optimization process.

Let us define a discrete scalar inner product of two discrete vectors $a^{n}=\left(a_{1}^{n}, \cdots, a_{K}^{n}\right)$ and $b^{n}=\left(b_{1}^{n}, \cdots, b_{K}^{n}\right)$ , $\mathrm{K}$ being the number of one-dimensional grid points, by a discrete summation over the time and space domains :

$$
<a^{n}, b^{n}>=\sum_{k=1}^{K} a_{k}^{n} b_{k}^{n}
$$

To simplify our presentation, we present the inverse problem with measurements data with only one thermocouple sensor, point $\mathrm{m}$ in the grid. Therefore, the first step in establishing a procedure for the solution of either inverse is thus the definition of an objective (cost) function: it is in our case a least squares performance index $J(p)$ that measures the difference between model predictions $T_{m}^{n}$ of temperature, given a heat flux parameter $p$ value, and measurements temperatures $\theta_{m}^{n}$, at point $m$ on the grid, time (n). The quadratic error or cost function $j(p)$, depending on the source parameters $p$, is defined by :

$$
J(p)=J \underbrace{\left(w^{1}(p), \ldots, w^{N}(p)\right)}_{\text {var iables } W}=\sum_{n=1}^{N}\left(T_{m}^{n}-\theta_{m}^{n}\right)^{2} \Delta t
$$


with $\theta_{m}^{n}$ the discrete measured temperature, at time n, point $\mathrm{m}$, and $T_{m}^{n}$ the discrete computed temperature vector, at time $n$, point $m$.

To minimize this quantity, by optimization algorithm, we need the derivatives of this least squares objective function $\mathrm{J}(\mathrm{p})$, with respect to the parameters $\mathrm{p}$.

\section{$\underline{\text { Adjoint and gradients computations }}$}

We introduce the adjoint state matrix $\varphi=\left(\varphi^{1 / 2} \cdots ; \varphi^{N+1 / 2}\right)$ adjoint of the direct state matrix $w, \varphi^{n+1 / 2}$ being a vector $(\mathrm{K}+1)^{*} 1$, for all $\mathrm{n}=0, \mathrm{~N}$. A Lagrangian formalism is used in the minimization of the functional $J(p)$ because the estimated dependent variable $w(p)$ appearing in such functional $J(p)$ needs to satisfy a constraint, which is the solution of the discrete direct problem. In order to derive the adjoint problem, the governing equation of the direct problem, is therefore multiplied by the Lagrange multiplier, integrated in the space and time domains of interest and added to the original cost functional $J(p)$. The following Lagrangian $L$ on these discrete quantities is:

$$
\begin{aligned}
& L(p, w, \varphi)=L(\underbrace{p^{1}, \ldots, p^{N}}_{\text {parameter } p}, \underbrace{w^{1}, \ldots, w^{N}}_{\text {var iables } w}, \underbrace{\varphi^{1 / 2}, \ldots, \varphi^{N+1 / 2}}_{\text {adjo int var iables } \varphi}) \\
& =\sum_{n=1}^{N}\left(T_{m}^{n}-\theta^{n}\right)^{2} \Delta t \\
& +\sum_{n=0}^{N-1}\left\langle\varphi^{n+1 / 2}, \frac{w^{n+1}-w^{n}}{\Delta t}-f\left(w^{n}\right)-(d f)\left(w^{n}\right)\left(w^{n+1}-w^{n}\right)\right\rangle
\end{aligned}
$$

Differentiating the Lagrangian $L$ with first order sensitivity variations, computing $\delta L$ as function of $\delta p, \delta w, \delta \varphi$, the variations of $\delta L$ with respect to $\delta w$ are cancelled with an adequate choice of the adjoint state $\varphi$. It leads to the discrete adjoint system ${ }^{31}$ in $\varphi^{n-1 / 2}$ unknown, $n$ going backward from $\mathrm{N}$ to 0 ,

$$
\begin{aligned}
& \frac{\varphi^{n-1 / 2}-\varphi^{n+1 / 2}}{\Delta t}=d f^{t}\left(w^{n-1}\right) \varphi^{n-1 / 2} \\
& +\left[\left(d^{2} f\right)\left(w^{n}\right)\left(w^{n+1}-w^{n}\right)\right] \varphi^{n+1 / 2}+2\left(T_{m}^{n}-\theta_{m}^{n}\right)^{2} \Delta t \\
& w^{N+1 / 2}=0 \quad N \geq n \geq 0
\end{aligned}
$$

With this particular choice of $\varphi$, the gradient of the cost function is simply obtained by :

$$
\nabla J=\frac{\partial J}{\partial p}=\frac{\partial L}{\partial p}
$$

Note that that $\left(d^{2} f\right)\left(w^{n}\right)$ is a tensor of dimension $(\mathrm{K}+1)^{*}(\mathrm{~K}+1)^{*}(\mathrm{~K}+1)$, and $\left[\left(d^{2} f\right)\left(w^{n}\right)\left(w^{n+1}-w^{n}\right)\right]$ is a square matrix $(\mathrm{K}+1)^{*}(\mathrm{~K}+1)$. We note also that the adjoint problem involves final conditions given instead of the initial conditions (direct problem): it has to be numerically solved by integrating backward in time as a terminal value problem. The final condition, not detailed here, simply results from the differentiation of (14).

The variations of $\delta L$ function of $\delta p$ leads to the expression of the discrete gradients:

$$
\frac{\partial J}{\partial p}=\sum_{n=0}^{N-1}\left\langle\varphi^{n+1 / 2},-\frac{\partial f}{\partial p}\left(w^{n}\right)-\frac{\partial d f}{\partial p}\left(w^{n}\right)\left(w^{n+1}-w^{n}\right)\right\rangle
$$

Note that $\frac{\partial f}{\partial p}\left(w^{n}\right)$ is a tensor $(\mathrm{K}+1)^{*} \mathrm{~N}, \frac{\partial d f}{\partial p}\left(w^{n}\right)$ is a tensor $(\mathrm{K}+1)^{*}(\mathrm{~K}+1)^{*} \mathrm{~N}, \frac{\partial J}{\partial p}$ is a vector $1 * \mathrm{~N}$. It can be shown $^{28}$ that gradients appeared as combination of direct and adjoint discrete quantities. We won't get into more detailed expressions, because the exact developed terms are quite complex and too big to be described here, the point being the method main principles and the corresponding applications.

\section{$\underline{\text { Optimization Minimization algorithm }}$}

Once the gradient of cost function is computed, we can now apply an iterative inverse procedure minimizing $J(p)$ to obtain an estimation of the unknown parameter optimal function $p_{\text {opt }}$. We will use the combination of a gradient steepest descent method at the beginning of minimization and a Quasi Newton method to finish the minimization.

The basic idea of the gradient Steepest Descent Method $^{32}$ is to move downwards on the objective function $J(p)$ along the direction of highest variation, in order to locate its minimum value. Therefore, the direction of descent is given by the gradient direction, since it is the one that gives the fastest increase of the objective function. Usually the steepest-descent method starts with large variations in the objective function and good initial exploration steps, but, as the minimum value is reached, the convergence rate becomes very low. The algorithm is (18) : 
- $p=q_{0}$ is the initial guess parameter, and $r$, the number of the optimizer iteration has the value $r=1, \ldots$, Nop, Nop being the maximum number of optimizer iteration.

- $d_{r}=\frac{\partial J}{\partial q_{r}}$ gives the descent direction

- $p=q_{r+1}=q_{r}+\mu_{r} d_{r}$ leads to $\mathrm{p}$ parameter updating with the descent coefficient $\mu_{r}$ chosen to satisfy the steepest descent of the $J(q)$ cost function $\mu_{r}=\operatorname{Inf}_{\mu} J\left(q_{r}-\mu d_{r}\right)$.

When steepest gradient method does not converge any more, the idea is to pursue the optimization process with a second order Quasi Newton method ${ }^{33,34}$, which has a strong local convergence. In these types of methods, the Hessian second derivative matrix is approximated in such a way that it does not involve the computation of costly second derivatives. Usually, the approximations for the Hessian are based on first derivatives (gradients) and it accelerates the convergence locally.

Starting with an initial guess for the estimated parameter $p=q_{0}$, and with an initial matrix $H_{0}^{-1}$ which is an approximation for the inverse of the Hessian, a Quasi-Newton Broyden Fletcher Goldfarb Shanno (BFGS) optimizer is used to update the parameter value $p=q_{r}$ at the optimizer step $r$, and the value of $H_{0}^{-1}$, until the number of total steps Nop of the optimizer is reached. We stops the process before if an optimal $p_{o p t}$ parameter is found, which causes the gradients to vanish (at least a local minimum of $\mathrm{J}(\mathrm{p})$ ). The BFGS algorithm is the following (19):

- $p=q_{0}, H_{r}=H_{0}$, are the initial guess parameter and Hessian, $r$ is again the current step of optimizer and $N_{o p}$ the total number of optimizer iterations.

- $d_{r}=-H_{r}^{-1} \frac{\partial J}{\partial q_{r}}$ gives the descent direction

- $\left.H_{r}=H_{r-1}+z\left(q_{r-1}, q_{r-2}, \frac{\partial J}{\partial q_{r}}, \frac{\partial J}{\partial q_{r-1}}, H_{r-1}\right)\right\}$

updates the Hessian approximated matrix, with $\mathrm{z}$ a function not explicited here

- $p=q_{r+1}=q_{r}-\mu_{r} d_{r}$ allows the parameter updating with the descent coefficient $\mu_{r}$ chosen to satisfy the steepest descent of the $J(q)$ cost function $\mu_{r}=\operatorname{Inf}_{\mu} J\left(q_{r}-\mu d_{r}\right)$.

\section{Inverse problem computation using automatic differentiation}

To compute numerically the adjoint and gradient discrete quantities for the inverse problem in heat convection coefficient, we have also used the Automatic Differentiation (AD) engine tool, Tapenade, developed at INRIA Sophia-Antipolis by the Tropics team ${ }^{24}$. Automatic differentiation is a family of techniques for computing the derivatives of a function defined by a computer program (interpreted as computing a mathematical function, including arbitrarily complex simulation codes), for sensitivity and gradient analysis applications ${ }^{35-37}$. The new program obtained is called the differentiated program. Automatic differentiation with adjoint models and gradients computations are used in many fields of science such as pioneering work in meteorology $\mathrm{y}^{38-40}$.

The derivatives of the instructions of a program (elemental operations) are combined according to the chain rule of differential calculus, leading to the two major modes of computing derivatives with $\mathrm{AD}$, the socalled forward (tangent-linear) mode and reverse (cotangent-linear or adjoint) mode.

- The forward mode uses directional derivatives on a given direction vector in the input space (tangent approach. It is appropriate to derive functions with small numbers of independent variables (input).

- The reverse mode uses derivatives starting with the dependent variables (output) and proceeding toward the independent variables (input), and it is computed in the reverse of the original program's order. It is appropriate for functions with small numbers of dependent variables (output) and lots of input independent variables. The reverse mode of automatic differentiation is functionally equivalent to hand written discrete adjoint codes.

The implementation of robust and effective automatic differentiation tools requires advances in compiler technology, graph algorithms, and automatic differentiation theory, and compared with other methods to compute adjoint and gradients, automatic differentiation offers a number of advantages:

- Accuracy: unlike finite difference approximations, derivatives computed via automatic differentiation exhibit no truncation error.

- Reduced software costs: automatic differentiation eliminates the time spent developing and debugging derivative code by hand, or experimenting with step sizes for finite difference approximations.

We have applied these techniques to our inverse thermal problem, considering that the flow of instructions in the direct program (Monopyro direct code), can be schematically represented as sequential instructions 
$(\text { Inst })^{n+1}$ to compute the direct state variables $w^{n+1}$ given the parameter $p$ $w^{n+1}=(\text { Inst })^{n+1}\left[w^{n}, w^{n-1}, \cdots \cdots, p, \cdots,\right], n=0, . ., N$

$(\text { Inst })^{n+1}$ are discrete functions (that could be non linear functions, recursive functions or interpolated tabulated functions) of discrete temperature and ablation variables. The final output of the program is the discrete cost function $J(p)=J(w(p))=J\left(\left(w^{1}, \ldots, w^{N}\right)(p)\right)$. The adjoint code in $\varphi$ variables is built by automatic backward differentiation of the output $J$ versus $w$ direct state variables, following and analyzing the flow of instructions in the direct program, and the dependences in $w$. The gradient computation of $J(p)$ versus $p$ parameter is built by automatic backward differentiation of the output $J(p)$ versus $p$ parameter, also following the flow of instructions in the direct program and analyzing the flow dependences in $p$. It can be shown again that the gradient result depends on the $w$ direct state variable and the $\varphi$ adjoint state variable.

\section{Numerical results}

We now present some applications of inverse problem of the estimation of time domain surface heat convection coefficient for thermally degradable material, on a onedimensional slab of thickness e, by using time domain temperature measurements taken below the boundary surface, at a given thermocouple position, during a time interval. As mentioned before, the inverse problem is formulated as a minimization problem involving an objective functional, through an optimization loop. We start the minimization loop by an initial guess on convection coefficient and try to restitute the measurements. In all the following curve results legends, INI stands for initial guess of the convection coefficient, NUM for reconstruction obtained at the end of optimization process, and OBS for the reference solution of the convection coefficient (when this targeted result is known) or for the corresponding measurements, input of inverse method. The final time is denoted by $t_{f}$.

We first define two test configurations on measurements:

- Synthetic measurements: the estimated temperatures are obtained from the solution of the direct problem, by using a given well known convection coefficient $\alpha_{0}(t)$. We want to restitute by inversion this coefficient.
- Noise measurements : the measurements may contain random errors, which are assumed here to be

- additive, uncorrelated, normally distributed, with zero mean and known standard deviation $(2 \%)$

○ additive, uncorrelated, uniformally distributed, with zero mean and known standard deviation (5\%)

Here, we want to see the effect of adding this noise to synthetic measurements on the reconstruction of convection coefficient $\alpha_{0}(t)$, in order to test the stability and robustness of the inverse method.

Moreover, we define now two similar quality estimators for inverse problem :

- A good estimator for the quality of restitution of temperature measurements is the $\mathrm{RMS}_{\mathrm{T}}$ error: Root Mean Square error between the $\theta_{m}^{n}$ measured temperature and the reconstructed temperature $\operatorname{Topt}_{m}^{n}$, at sensor $\mathrm{m}$, for the optimal inverse solution $p_{\text {opt }}$ :

$$
R M S_{T}=E Q M=\sqrt{\frac{\sum_{n=1}^{N}\left(\text { Topt }_{m}^{n}-\theta_{m}^{n}\right)^{2}}{N}}
$$

- A good estimator for the quality of restitution/identification of convection coefficient is the $\mathrm{RMS}_{\mathrm{p}}$ error between the reference $\alpha_{0}$ convection coefficient and the reconstructed optimal $p_{\text {opt }}$ :

$$
R M S_{p}=\sqrt{\frac{\sum_{n=1}^{N}\left(p_{o p t}^{n}-\alpha_{0}{ }^{n}\right)^{2}}{N}}
$$

Theses tests have been realized to address the problem of fluxes identification on a carbon/resin material. To ensure the method, we first tried to examine the effects of pyrolysis (test 1) and ablation (test 2) separately, then we worked on the real ablating and pyrolysing material (test 3 ), then we applied the new method to operationnal cases, such as the quite well known ARD (Atmospheric Reentry Demonstrator, test 4 with a different material: aleastrasil), or the more relevant arc plasma torch test on the considered carbon/resin material, where the fluxes are very high and the flow conditions better known and where some fluxmeters measurements are also available (test 5).

\section{Test 1 : Identification of virgin material without ablation, $x 0=1.3 \mathrm{~mm}$}

We use synthetic data (errorless measurements). We start (INI) with a bad initial guess half value of convection coefficient, with sharp discontinuity. Fig. 17 shows a good agreement for the reconstruction (NUM) of 
the convection coefficient, compared to the reference convection coefficient (OBS), with the inverse code developed in section III ("hand computed" gradients and adjoints), except near the final time. The RMS error on the flux is 0.04 .

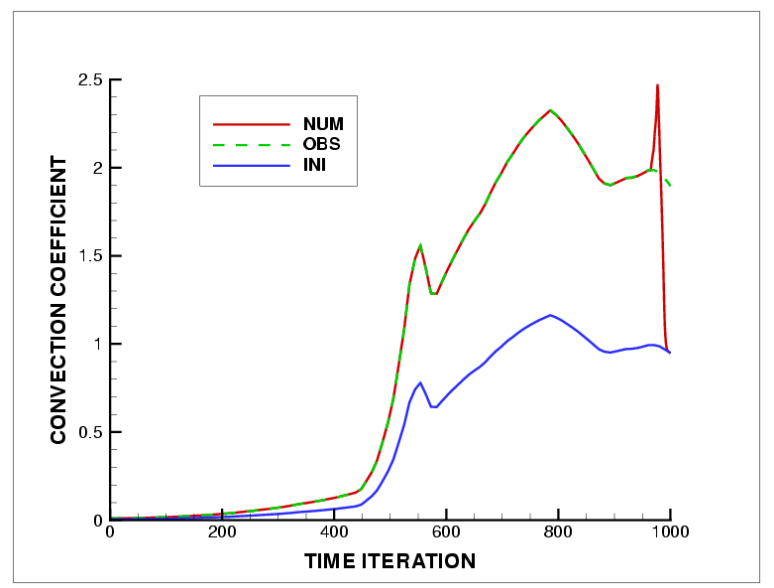

Figure 17. Test 1 : Flux Identification of virgin material without ablation , $x 0=1.3 \mathrm{~mm}$

The results shown in Fig. 18 were obtained with the inverse code developed in section IV (Automatic Differentiation tool was used) and are very correct too. Near final time, the value of the estimated flux has very little influence on the temperature in the material, at $\mathrm{x} 0$. Even if the flux is worse evaluated at the end, the impact on the corresponding solution is not visible.

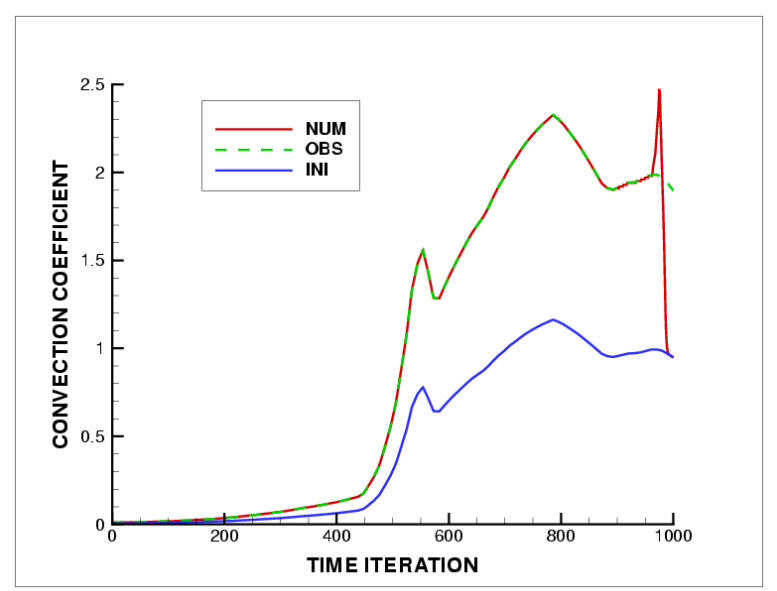

Figure 18. Test 1 : Flux Identification of virgin material without ablation, $x 0=1.3 \mathrm{~mm}$ Automatic Differentiation tool
Fig. 19 shows that the RMS error on temperature obtained at the end of optimization process (also using the Automatic Differentiation tool), is very low (0.01), and we can observe the change in optimizer (iteration 25), switching from gradient steepest descent at the beginning, to Quasi Newton after. The gain in convergence is promising, after 60 optimizer iterations.

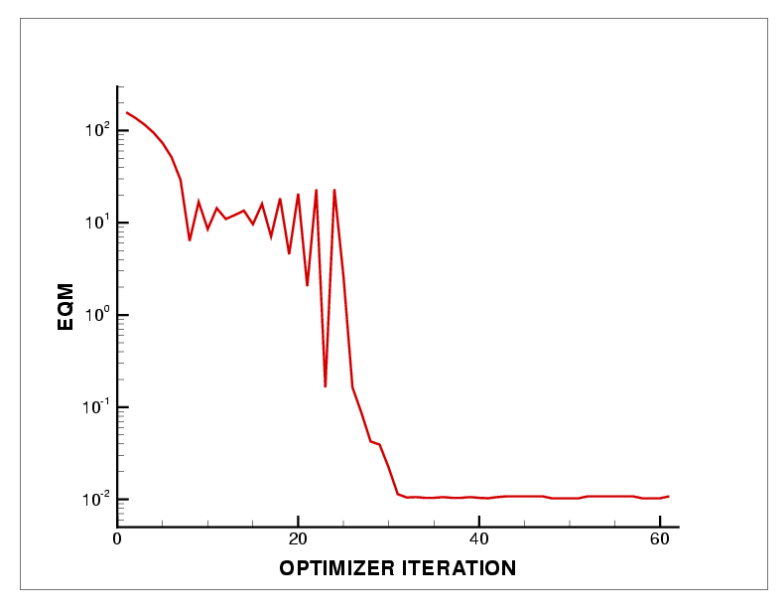

Figure 19. Test 1 : Temperature RMS error Virgin material without ablation , $x 0=1.3 \mathrm{~mm}$ Automatic Differentiation tool

Test 2 : Identification of High Flux with ablation, Carbon/Resin material , $\mathrm{x} 0=\mathbf{2 . 6} \mathrm{mm}$

It is a quite difficult test case, with high fluxes. In Fig. 20, a good agreement in the reconstructed convection coefficient value is obtained, except at final time, with initial half guess and using synthetic data (errorless measurements). The RMS error on the flux is 0.06 . 


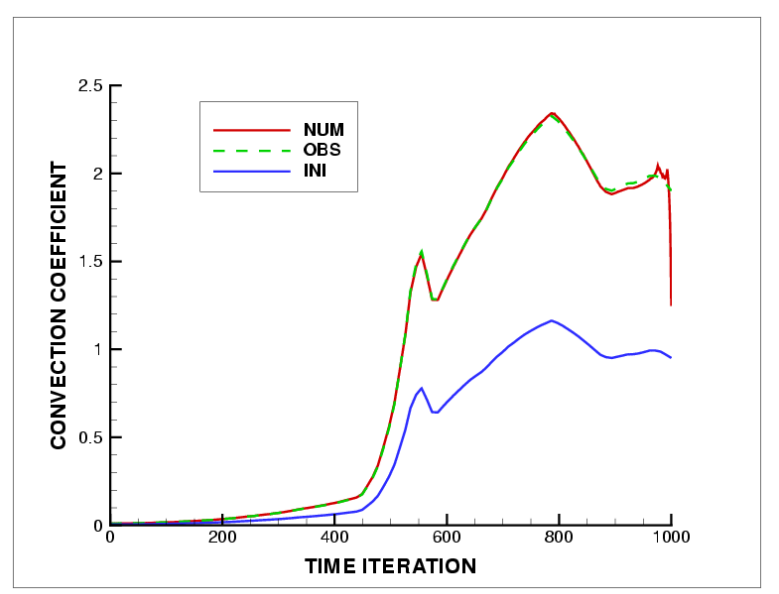

Figure 20. Test 2 : Identification of High Flux with ablation, $x 0=2.6 \mathrm{~mm}$

Fig. 21 shows that the RMS error on measured temperature obtained at the end of optimization process is very low (0.7), after 70 optimizer iterations.

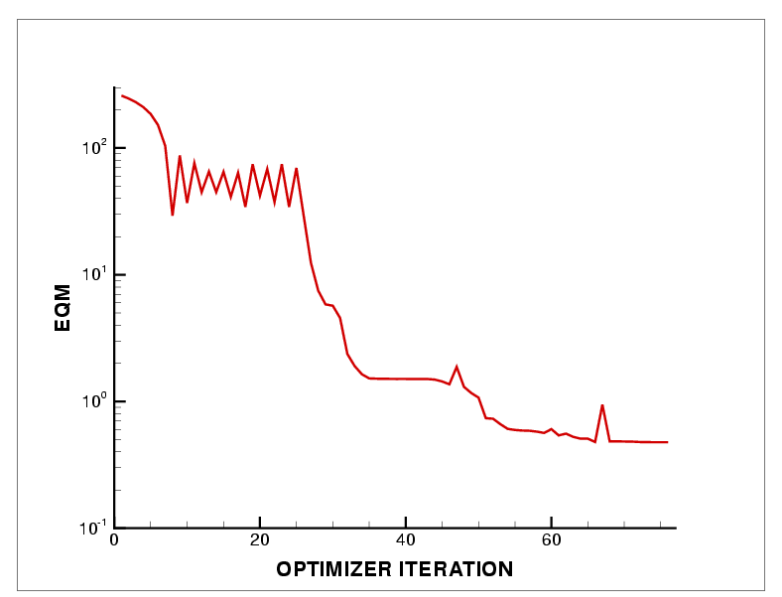

\section{Figure 21. Test 2 : RMS (EQM) error on temperatures}

Test case with ablation , $x 0=2.6 \mathrm{~mm}$

Fig. 22 shows results in the convection coefficient obtained, with initial half of the value, additive, uncorrelated, normally distributed, zero mean and known standard deviation $(2 \%)$ noise. The RMS error on the flux is 0.105 , which is satisfactory.

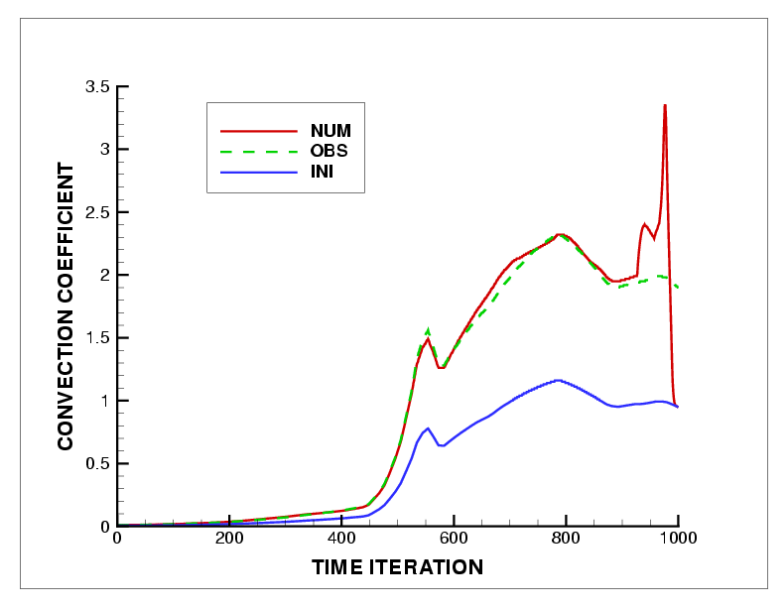

Figure 22. Test 2 : Identification of High Flux with ablation, with $2 \%$ normal noise , $x 0=2.6 \mathrm{~mm}$

Fig. 23 shows results in the convection coefficient obtained, with initial half of the value, additive, uncorrelated, uniformally distributed, zero mean and known standard deviation (5\%) noise. The RMS error on the flux is 0.125 .

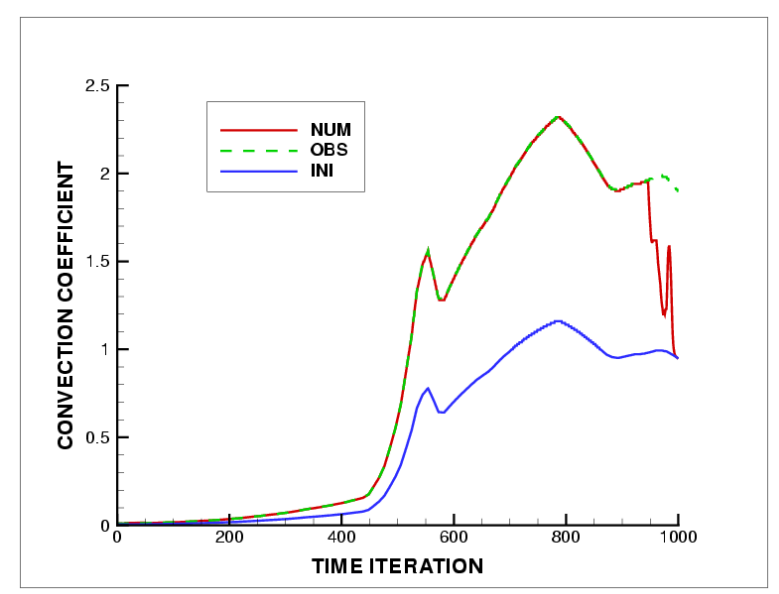

Figure 23. Test 2 : Identification of High Flux with ablation, with $5 \%$ uniform noise, $x 0=2.6 \mathrm{~mm}$

Test 3 : Identification of High Flux with ablation and pyrolysis, Carbon/Resin material $x 0=4.2 \mathrm{~mm}$

We now examine the present inverse analysis approach for a difficult test case, with high fluxes, ablation, pyrolysis, and deep thermocouples location and synthetic measurements on a "real" material. 
Successful results are obtained in the reconstructed convection coefficient and displayed on Fig. 24, with a RMS error on the flux of 0.07 .

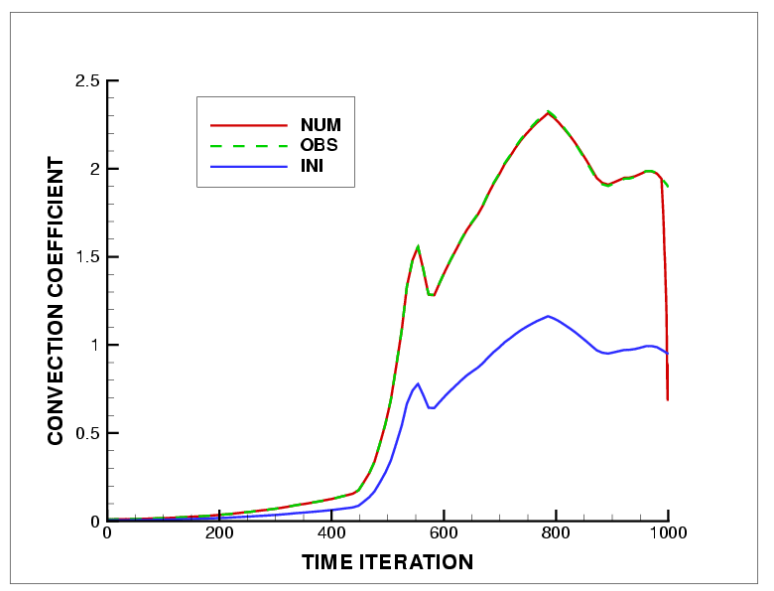

Figure 24. Test 3 : Identification of High Flux with ablation and pyrolysis, $x 0=4.2 \mathrm{~mm}$

Fig. 25 shows that the RMS error on temperature at the sensors obtained at the end of optimization process is very low (0.9), after 75 optimizer iterations.

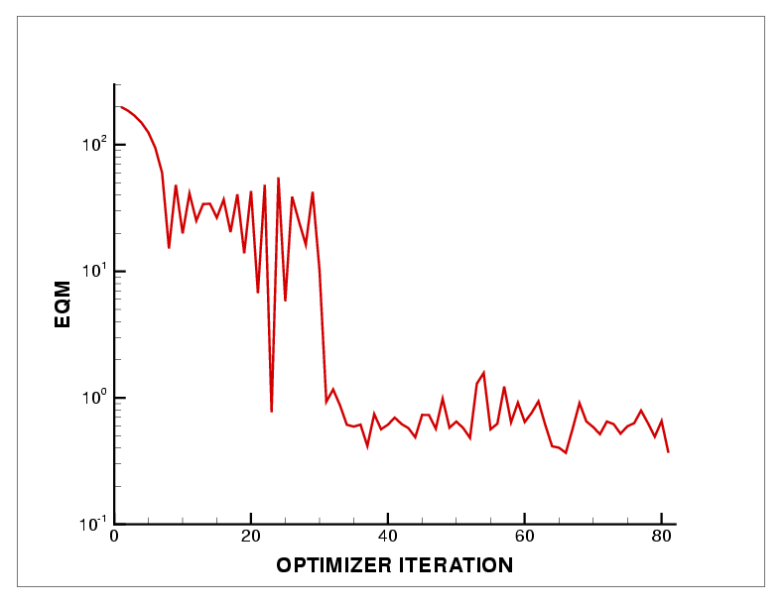

Figure 25. Test 3 : Temperature RMS (EQM) error High Flux with ablation and pyrolysis, $x 0=4.2 \mathrm{~mm}$

\section{Test 4 : ARD Test case}

We now examine the present inverse analysis approach for the ARD flight test case. The Atmospheric Reentry Demonstrator (ARD) was a suborbital reentry test flown on the third Ariane 5 flight. ARD was launched in october 1998 from Kourou, French Guyana, by an
Ariane 5 and splashed down 1 hour $41 \mathrm{~min}$. after liftoff. It was recovered and transported in EADS Astrium's Aquitaine plant for expertise. More than 200 different parameters were recorded during flight. After ARD recovery, a preliminary analysis of recorded data has been performed.

A picture of the recuperation of the capsule is given on Fig. 26. The heat shield (Fig. 27) has been expertised (Fig. 28) after the flight.

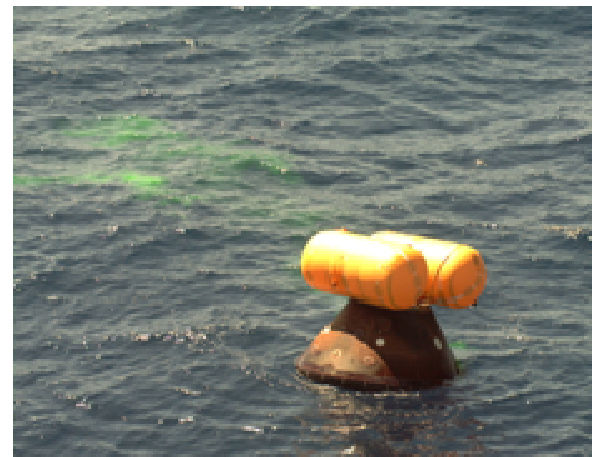

Figure 26. ARD sea landing

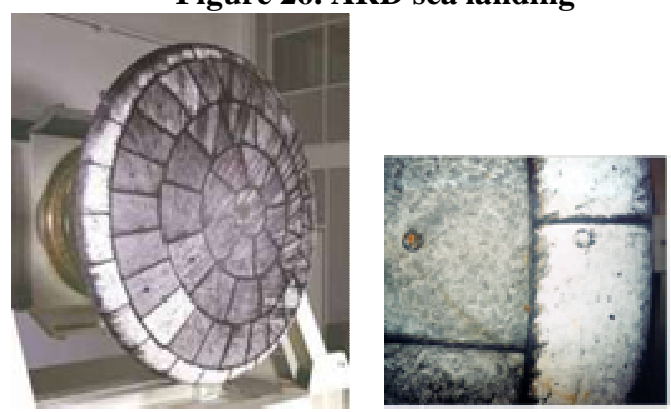

Figure 27. ARD heat shield

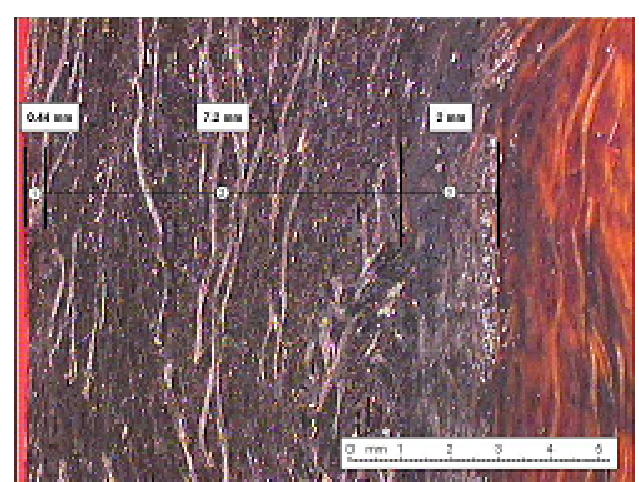

Figure 28. ARD thermocoil

Successful results are obtained in the reconstructed flux (Fig. 29), which are very similar to those obtained before (see Fig. 30 and ${ }^{23}$ ). 


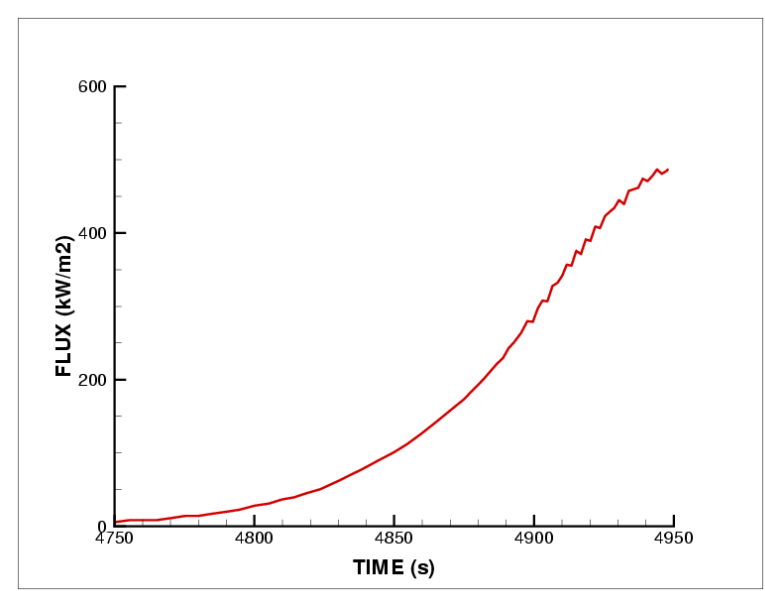

Figure 29. ARD heat flux restitution

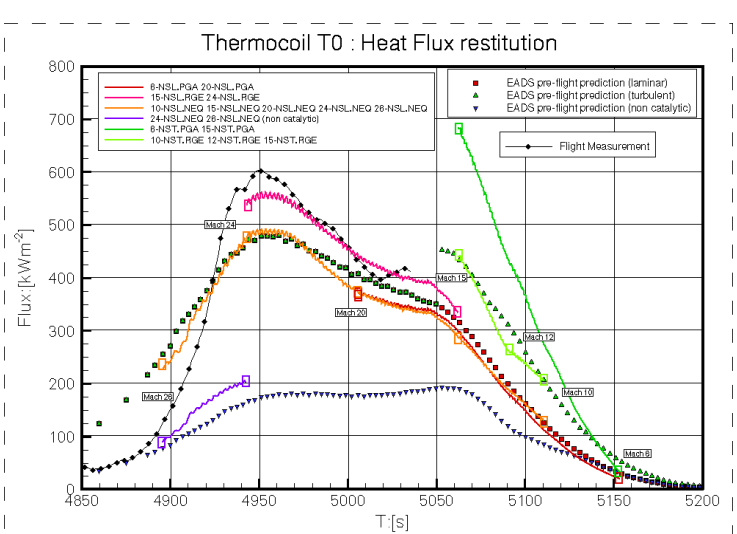

Figure 30. ARD post flight analysis : heat fluxes, courtesy of ${ }^{23}$

\section{Test 5 : Operational test case (Plasma Jet case)}

This case has been investigated to improve the robustness on an industrial problem where many experimental data were available. The industrial applications are straight forward. The plasma jet facility of the Astrium's Aquitaine plant is shown on Fig. 15, with the schematic principal of a plasma torch. The experimental test facility uses four coupled plasma torchs.

The tested material is equipped with eight thermocouples at two stations in the duct.

This case is a good industrial application where the method can be used. The robustness of the model can be tested in a concrete situation with direct industrial impacts. A set of experimental data is available in tests made in the plasma torch. This experimental test facility is located at Astrium Aquitaine site and Figure 31 presents an overview of the torch and a schematic drawing of a torch.
Four plasma torches are actually coupled to an electric power of $20 \mathrm{MW}$ and debit in a mixing chamber, the air plasma is then relaxed in a nozzle

In the output nozzle, the behaviour of some thermal protection can be tested, as on the views proposed Figures 9 and 10. It is also possible to follow the nozzle by a rectangular duct section, which of one movable wall can accommodate a specimen sample for testing a thermal protection material, in this case a composite carbon / resin. The test material is equipped with two sections of 4 thermocouples each, as suggested by Figure 32 . The ablation of the sample is compensated during the test, and the test set up is equipped with a pyrometer to access the surface temperature, a laser impact providing an ablation measurement, and two fluxmeters located on the wall of the duct, in front of the sample specimen. The optical measurements are done through an hublot locally cooled ${ }^{7}$.
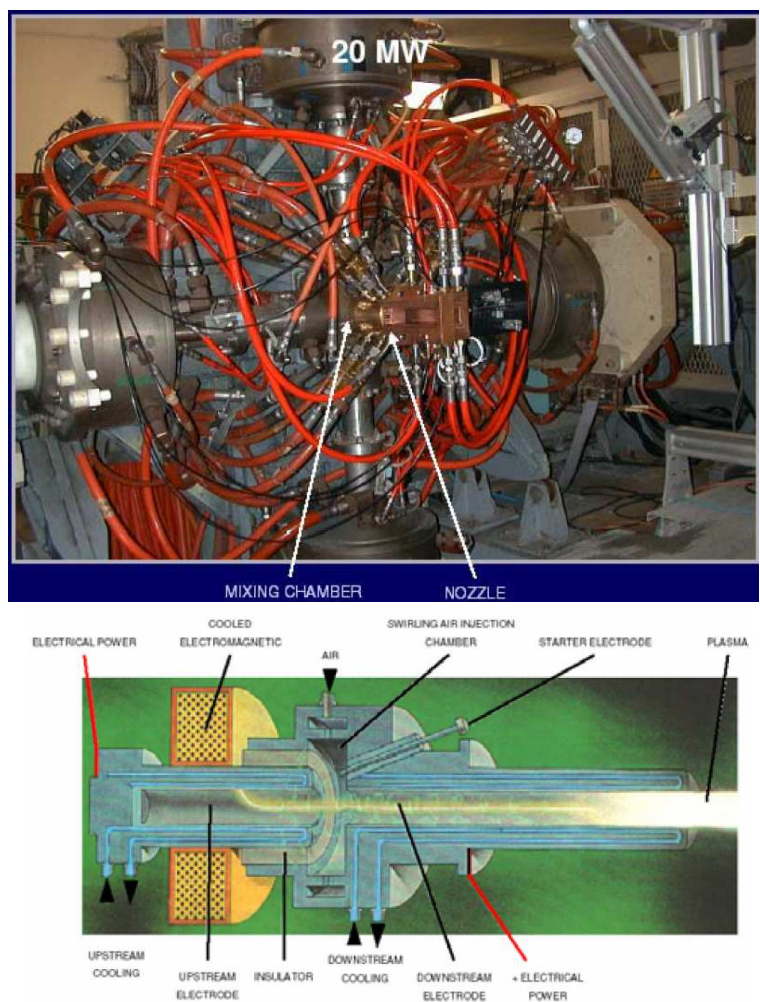

Figure 31. Plasma jet facility

\section{Figure 32 : Plasma Jet - Measurements principles}

A simulation of Navier-Stokes type, as shown Figure 33 (pressure) yields a first estimate of flux at the surface of the tested material. In this case, simulation provides an heat flux of approximately $12-13 \mathrm{~mW} / \mathrm{m} 2$ on the specimen. 


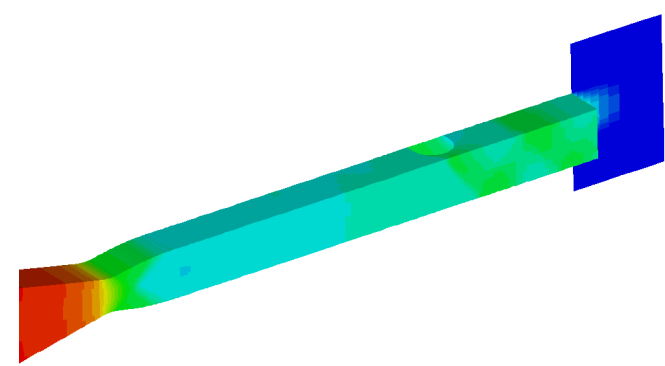

Figure 33: Plasma Jet - Navier-Stokes Simulation of the flow

On the test run here, we have 8 of thermocouples, which we treat by inverse method independently of each other to restore the heat flux at the surface of the specimen. Figure 34 provides a comparison between 8 temperatures measured and 8 temperatures restituted at the end of the optimization process.

Figure 35 provides a comparison between the ablation measurement with laser impact and restituted ablations. As expected, thermocouples located closer to the surface are those which give the best results.

The mean squared differences obtained at the end of the optimization is around $20 \mathrm{~K}$, which is correct in relation to temperatures reached, significant variances in temperature corresponds more to the cooling phase (specific to the test, because in-flight cooling is rare), the phase for which direct thermal model remains imperfect.

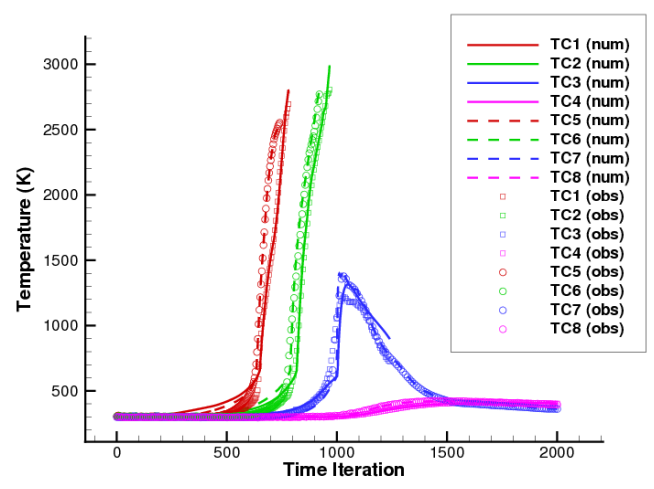

Figure 34, Test 5.2: simulated (num) and measured (obs) levels of temperatures at the 8 sensors.

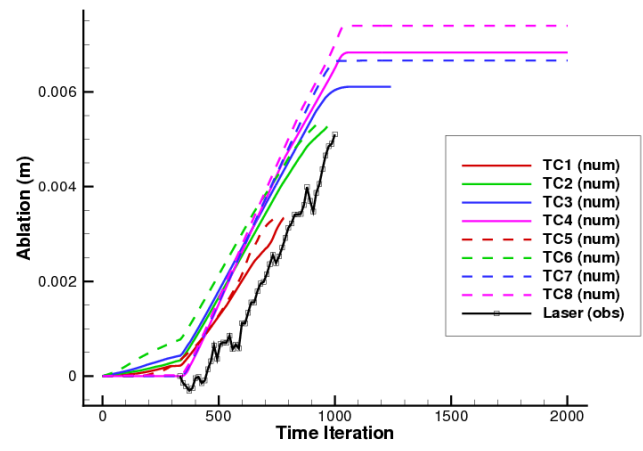

Figure 35, Test 5.2: simulated (num) and measured (Laser Obs) levels of ablation.

To compare to other measurements (pyrometer and fluxmeter) made during the test with the restitution, it is necessary to correct these measurements to take into account the radiative effects in the test medium.

Indeed, as regards the fluxmeters (which are also the subject of a program of improving measurements at high fluxes), the specimen heated emits radiation which impact on the flux levels measured in front of the specimen. Regarding the pyrometer, it is necessary to make an assumption about the emissivity of the material, estimated at $0.85 \pm 0.05$.

Figure 36 compares the surface temperature provided by the corrected pyrometer measurements to the wall temperatures restituted by computation, for each of the 8 measurements examined.

Figure 37 compares the heat flux returned by the inverse method to the corrected flux measurements, the radiation flux on the fluxmeters being about $7 \mathrm{MW} / \mathrm{m}^{2}$.

The results are satisfactory, especially for the sensors closest to the wall, which remain less sensitive to defects in thermal modeling or resolution of the direct method. The restituted fluxes are also consistent with the NavierStokes simulations performed on this case.

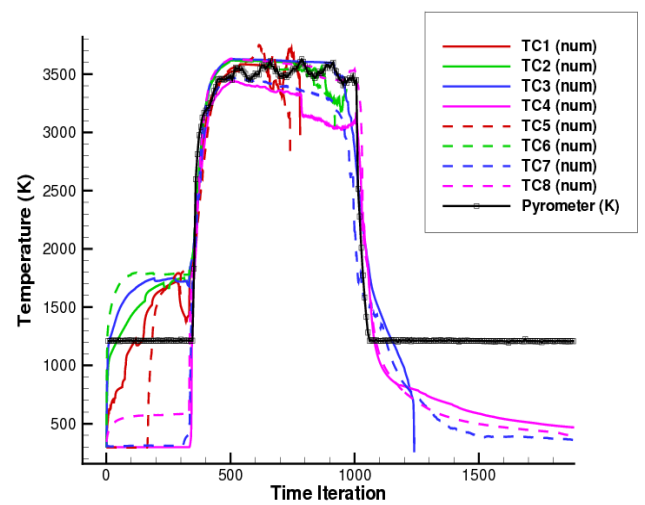


Figure 36, Test 5.2: simulated (Temp) and measured (Pyrometer) levels of surface temperatures.

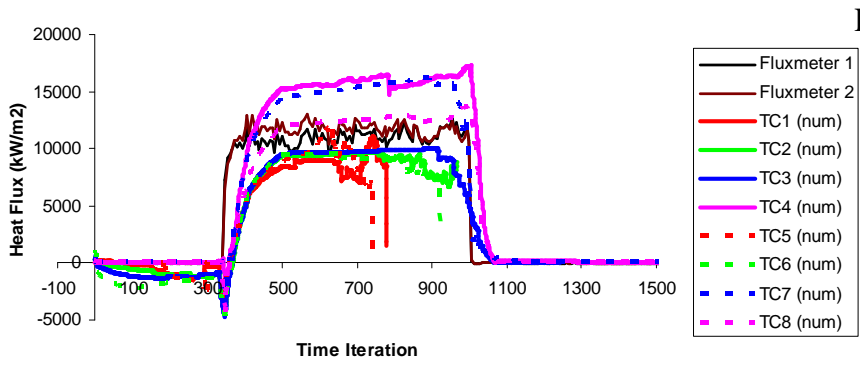

Figure 37, Test 5.2: simulated (Flux) and measured (Fluxmeter) levels of heat fluxes.

\section{Conclusion}

Motivated by atmospheric re-entry of aerospace vehicles and Thermal Protection System dimensioning problems, this paper is concerned with inverse analyses of highly dynamical heat fluxes. It addresses the inverse problem of using temperature measurements to estimate the heat flux convection coefficient, at the surface of ablating materials.

The inverse problem is formulated as a minimization problem involving a least square problem functional, through an optimization loop. An optimal control formulation (Lagrangian, adjoint and gradient computations) is then applied and developed, using an inverse software Monopyro which was developed at EADS Astrium Les Mureaux, and which is a transient one-dimensional thermal code, with ablative surface and Gear integration scheme.

Several validation test cases, using synthetic, noisy on-ground and in-flight data temperatures measurements are carried out, by applying the results of the minimization algorithm. Main results are:

- Validity of the inverse formulation for the description of the temperature and ablation variables evolution

- Optimization improvement by using a combined gradient steepest descent method at the beginning of minimization process and Quasi Newton method to finish the minimization,

- Convection coefficient restitution has been improved for hard cases (with great ablation) for fluxes functions containing sharp corners and discontinuities,

- Successful test case on carbon/resin material with high heat fluxes and large magnitudes, ablation including pyrolysis effects, and operational data (such as ARD test case, and jet plasma test case), even if there are some experimental and model errors in the direct thermal code Monopyro.

- Encouraging results with an automatic differentiation tool are also obtained, without ablation

Future works have to be done on the:

- Improvement of the direct Monopyro model, to better take into account the ablation, pyrolysis effects, ...

- Robustness to initial guess, sensitivity to measurements, number and position of sensors, and application of regularization methods to stabilize noise errors on measurements,

- Implementation of the automatic differentiation tool to generate the inverse code,

- Thermal model uncertainties influences on the accuracy of extracted flight heat flux, athermanous enthalpy identification,

- Validations on aerothermal flight measurements.

\section{Acknowledgments}

This work was sponsored by EADS Astrium. The authors would like to acknowledge Benoit Fourure, Laurent Fusade, Antonio Rivas, Jacques Soler, Philippe Tran, (EADS Astrium) and Eric Duceau, Isabelle Terrasse, (EADS-IW) for their efficient support.

\section{References}

\footnotetext{
${ }^{1}$ Macret, J.L., Leveugle, T., "The Atmospheric Reentry Demonstrator program : overview and flight results", IAF-99V.2.10, 50th International Astronautical Congress, 4-8 Oct 1999/Amsterdam, The Netherlands.

${ }^{2}$ Macret, J.L., Paulat, J.C., Tran, P., Rolland, J.Y., and Steinkopf, M. "Post Analysis of the atmospheric reentry demonstrator flight" 51th International Astronautical Congress IAF-00-V.2.05s, Rio de Janeiro, Brazil, October 2000.

${ }^{3}$ Boukhobza, P., Paulat, J.C., Riccardi, S., Soler, J.,Tran, P., Véronneau, Y., and Walpot, L.,"Recent re-entry flight experiments lessons learned - ARD”, Flight Experiments for Hypersonic vehicle development, Von Karman Institute for Fluid Dynamics, 24 - 27 October 2005.

${ }^{4}$ Epherre, J.F. and Laborde, L. "Pyrolysis of carbon phenolic composites", Fourth International Symposium Atmospheric Reentry Vehicles and Systems - Arcachon - 21 au 23 mars 2005.

${ }^{5}$ Velghe, A. Duffa, Nguyen-Bui, N-T-H., and Dubroca, B., "Modeling of the Surface State of Ablatable Material by Reaction-Diffusion Phenomenon", AIAA/CIRA 13th International Space Planes and Hypersonics Systems and Technologies Conference, 16 - 20 May 2005 Centro Italiano Ricerche Aerospaziali (CIRA), Capua, Italy, 2005.

${ }^{6}$ Bouilly, J-M., Dariol, L. and Leleu, F. "Ablative Thermal Protections for Atmospheric Entry. An Overview of Past Missions and Needs for Future Programmes", 5 thEuropean Workshop Thermal Protection Systems and Hot Structures, 17/19 May 2006 Noordwijk, The Netherlands.
} 
${ }^{7}$ Boursereau, F., Donnart, P., Bouffet, S., Astier, JC, Jullien P., and Foltyn, M. "Theoretical and experimental investigations on a new configuration arc plasma torch", 6st International Symposium - Atmospheric Reentry Vehicles and Systems ; Arcachon, France, 21 au 23 mars 2005.

${ }^{8}$ Dubroca, B., Duffa, G., and Leroy, B. "Heterogeneous Reactions on TPS Surfaces: General Derivation and Equilibrium Limit“, AIAA/CIRA 13th International Space Planes and Hypersonics Systems and Technologies Conference, 16 - 20 May 2005 Centro Italiano Ricerche Aerospaziali (CIRA), Capua, Italy, 2005.

${ }^{9}$ Cosson, A.E., Thivet, F., Soler, J., Tran, P., Spel, M., Dieudonné, W., Paulat, J.C., Parmpolini, M., and Moulin, J., "Pre-X Aerothermodynamics Implications at System Level", Fourth International Symposium Atmospheric Reentry Vehicles and Systems - Arcachon - 21- 23 mars 2005.

${ }^{10}$ Wright, M., Milos, F., and Tran, P.”Survey of Afterbody Aeroheating Flight Data for Planetary Probe Thermal Protection System Design “, 38th AIAA Thermophysics Conference, June of 2005 in Toronto, Ontario Canada.

11 Bouilly, J-M., "Thermal Protection System of the Huygens Probe during Titan Entry: Flight Preparation and Lessons Learned", 5 thEuropean Workshop Thermal Protection Systems and Hot Structures, 17/19 May 2006 Noordwijk, The Netherlands.

${ }^{12}$ Collinet, J., Brenner, P., and Palerm, S., "Dynamic stability of the HUYGENS probe at Mach 2.5" , Aerospace Science and Technology Volume 11, Issues 2-3, March-April 2007, Pages 202-210.

${ }^{13}$ Beck J.V., B Blackwell, B., Inverse Heat Conduction: IllPosed Problems, CR St Clair - A Wiley \{Interscience Publication, New York, 1985.

${ }^{14}$ Machado, H.A. and Orlande H.R.B. "Inverse analysis for estimating the timewise and spacewise variation of the wall heat flux in a parallel plate channel", International Journal of Numerical Methods for Heat \& Fluid Flow, 1997.

${ }^{15}$ Walker, D.G. and Scott, E.P. “'A Method for Improving Two-Dimensional High Heat Flux Estimates from Surface Temperature Measurements," proceedings of the 32nd AIAA Thermophysics Conference, AIAA-97-2574, Atlanta, GA, June 1997.

${ }^{16}$ Li H. Y. and Yan, W. M., "Estimation of Wall Heat Flux in an Inverse Convection Problem," AIAA Journal of Thermophysics and Heat Transfre, Vol. 13, No. 3, pp. 394-396, 1999.

${ }^{17}$ Blanc, G., Beck J.V., and Raynaud, M., " Solution of the inverse heat conduction problem with a time variable number of future temperatures ", Numerical Heat Transfer, Part B, Vol. 32, pp.437-451,1997.

${ }^{18}$ Hoornaert, A., Pelissier, C., Le Sant, Y., Thivet, F., Millan, P., "On-ground validation of rear-face thermography to measure surface heat fluxes in hypersonic flows", 6th International Symposium on Launchers Technologies - Munich (Germany) - 8 au 11 nov. 2005.

${ }^{19}$ Lions, J.L., Contrôle Optimal des Systèmes gouvernés par des équations aux Dérivées Partielles, Dunod Paris, 1968.

${ }^{20}$ Alestra, S., Terrasse, I. and Troclet, B., "Inverse Method for Identification of Acoustic Sources at Launch Vehicle Liftoff", AIAA Journal, Vol 41, Number 10, pages 1980- 1987, October 2003.

${ }^{21}$ Troclet, B, Alestra S., Terrasse, I. Jeanjean, S., and Srithammavanh, V., "Identification of Overpressure Sources at Launch Vehicle Liftoff Using an Inverse Method", AIAA
Journal of Spacecraft and Rockets 2007 0022-4650 vol.44 no.3 (597-606).

${ }^{22}$ Rivas, A., "Monopyro, dépouillement des thermobobines : Monopyro software équations", Aerospatiale Technical Internal note, 2000.

${ }^{23}$ Tran, P., and Soler, J. "Atmospheric reentry demonstrator, Post-Flight Analysis : Aerothermal Environment", 2nd International Symposium - Atmospheric Reentry Vehicles and Systems ; Arcachon - March 2001.

${ }^{24}$ Hascoët L., Greborio, R.M., and Pascual V., "Computing Adjoints by Automatic Differentiation with Tapenade ", Ecole CEA-EDF-INRIA "Problèmes non-linéaires appliqués", Paris, 2002.

${ }^{25}$ Dubois, F., and Rivas, A. "Volumes finis à l'Aérospatiale", Ecole CEA EDF-INRIA "Méthodes de Volumes Finis", 28 - 30 October 1992.

${ }^{26}$ Bouffet, S. "Document Plan Logiciel : Software Guide Documentation Code BE13" LY211 n ${ }^{\circ} 138717$, EADS LV Technical Internal note, 2002.

${ }^{27}$ Roache, P. J., Computational fluid dynamics, Hermosa publishers, 1972, PO Box 8172, Albuquerque.

${ }^{28}$ Collinet, J "Monopyro Technical report on High fluxes results with a new inverse formulation for ablative material" EADS Astrium Technical Internal note, 2007.

${ }^{29}$ Dubois, F., "A Sparse matrix solver for Monopyro inverse code", EADS Technical Internal note, May, 2007.

${ }^{30}$ Bamberger, A., Chavent, G. and Lailly, P., "About the Stability of the inverse problem in 1D wave equation", Journal of Applied Mathematics and Optimisation, 5:1-47, 1979.

${ }^{31}$ Tekitek, M.M., Bouzidi, M., Dubois, F., and Lallemand, P., "Adjoint lattice Boltzmann equation for parameter identification", Computers \& Fluids, 35 (8-9), 805-813, 2006.

${ }^{32}$ Minoux, M., Mathematical Programming: Theory and Algorithms, Chichester: John Wiley and Sons, 1986.

${ }^{33}$ Gilbert, J.Ch., and C.Lemaréchal, C., "Some numerical experiments with variable-storage quasi-Newton algorithms". Mathematical Programming, 45 (1989), pp. 407-435.

34 Bonnans, J.F., Gilbert, J.Ch., Lemaréchal, C., Sagastizabal, C., Numerical Optimization Theoretical and Practical Aspects, Springer Verlag, Berlin. (2002).

35 Griewank, A., "Achieving Logarithmic Growth of Temporal and Spatial Complexity in Reverse Automatic Differentiation", Optimization Methods and Software, 1(1):3554, 1992.

${ }^{36}$ Faure, C., "Quelques aspects de la simplification en calcul formel", Thèse de l'Université de Nice-Sophia Antipolis, 1992.

37 Faure, C., and Papegay, Y.,"Odyssée User's Guide Version 1.7”, Rapport technique, INRIA, 1998, no 224 URL: http://www.inria.fr/rrrt/rt-0224.html.

${ }^{38}$ Le Dimet, F., and Talagrand, O., "Variational algorithms for analysis and assimilation of meteorological observations : theoretical aspects", in: Tellus, 1986, vol. 38A, p. 97-110.

${ }^{39}$ Talagrand, O.and Courtier, P., "Variational assimilation of meteorological observations with the adjoint vorticity equation. I: Theory", Q. J. R. Meteorol. Soc. 1987, vol. 113, no 478, pp. 1311-1328.

${ }^{40}$ Talagrand, O., "The use of adjoint equations in numerical modelling of the atmospheric circulation", Automatic Differentiation of Algorithms : Theory, Implementation and Application, A. Griewank, G. Corliss (editors), SIAM, 1991, p. 169-180. 